\title{
THE BOLOCAM GALACTIC PLANE SURVEY. II. CATALOG OF THE IMAGE DATA
}

\author{
Erik Rosolowsky ${ }^{1}$, Miranda K. Dunham ${ }^{2}$, Adam Ginsburg ${ }^{3}$, E. Todd Bradley ${ }^{4}$, James Aguirre $^{5}$, John Bally ${ }^{3}$, \\ Cara Battersbi ${ }^{3}$, Claudia Cyganowski ${ }^{6}$, Darren Dowell ${ }^{7}$, Meredith Drosback ${ }^{8}$, Neal J. Evans II $^{2}$, Jason $^{3}$ GlenN $^{3}$, \\ Paul Harver $^{2,3}$, Guy S. Stringfellow ${ }^{3}$, Josh Walawender ${ }^{9}$, and Jonathan P. Williams ${ }^{10}$ \\ ${ }^{1}$ Department of Physics and Astronomy, University of British Columbia Okanagan, 3333 University Way, Kelowna BC, V1V 1V7, Canada; erik.rosolowsky@ @bc.ca \\ ${ }^{2}$ Department of Astronomy, University of Texas, 1 University Station C1400, Austin, TX 78712, USA \\ ${ }^{3}$ CASA, University of Colorado, 389-UCB, Boulder, CO 80309, USA \\ ${ }^{4}$ Department of Physics, University of Central Florida, Orlando, FL 32816, USA \\ ${ }^{5}$ Department of Physics and Astronomy, University of Pennsylvania, Philadelphia, PA 19104, USA \\ ${ }^{6}$ Department of Astronomy, University of Wisconsin, Madison, WI 53706, USA \\ ${ }^{7}$ Jet Propulsion Laboratory, California Institute of Technology, 4800 Oak Grove Dr., Pasadena, CA 91104, USA \\ ${ }^{8}$ Department of Astronomy, University of Virginia, P.O. Box 400325, Charlottesville, VA 22904, USA \\ ${ }^{9}$ Institute for Astronomy, 640 N. Aohoku Pl., Hilo, HI 96720, USA \\ ${ }^{10}$ Institute for Astronomy, 2680 Woodlawn Drive, Honolulu, HI 96822, USA \\ Received 2009 May 30; accepted 2010 March 18; published 2010 April 22
}

\begin{abstract}
We present a catalog of 8358 sources extracted from images produced by the Bolocam Galactic Plane Survey (BGPS). The BGPS is a survey of the millimeter dust continuum emission from the northern Galactic plane. The catalog sources are extracted using a custom algorithm, Bolocat, which was designed specifically to identify and characterize objects in the large-area maps generated from the Bolocam instrument. The catalog products are designed to facilitate follow-up observations of these relatively unstudied objects. The catalog is $98 \%$ complete from $0.4 \mathrm{Jy}$ to $60 \mathrm{Jy}$ over all object sizes for which the survey is sensitive $(<3.5)$. We find that the sources extracted can best be described as molecular clumps-large dense regions in molecular clouds linked to cluster formation. We find that the flux density distribution of sources follows a power law with $d N / d S \propto S^{-2.4 \pm 0.1}$ and that the mean Galactic latitude for sources is significantly below the midplane: $\langle b\rangle=(-0.095 \pm 0.001)$.
\end{abstract}

Key words: catalogs - dust, extinction - Galaxy: structure - radio continuum: ISM - stars: formation

Online-only material: color figures

\section{INTRODUCTION}

The advent of large-number bolometer arrays at good observing sites has enabled the mapping of large sections of the sky in the millimeter continuum. These observations are ideally suited for unbiased searches for dense gas traced in dust thermal continuum emission. Even at the cold temperatures and high densities found in molecular clouds, dust emission remains an optically thin tracer of the gas column density. Despite some uncertainties in the properties of dust emission in these regimes, these observations represent the most efficient way to detect high column density features in the molecular interstellar medium (ISM). The original surveys of (sub)millimeter continuum emission focused on nearby molecular clouds (e.g., Motte et al. 1998; Johnstone et al. 2000; Enoch et al. 2007). With improvements in instrumentation and methodology, surveys of large sections of the Galactic plane have become possible. This paper presents results from one such survey: the Bolocam Galactic Plane Survey (BGPS). The Bolocam instrument at the Caltech Submillimeter Observatory has been used to conduct a survey of the Galactic plane from $-10^{\circ}<\ell<90.5$ and $-0.5<b<0.5$ in the continuum at $\lambda=1.1 \mathrm{~mm}$. The survey has been augmented with larger latitude coverage from $77^{\circ}<\ell<90.5$ and studies of individual star forming regions near $\ell=111^{\circ}$ and in the W3/4/5, Gem OB1, and IC 1396 regions. The observations, reduction and calibration of survey data are presented in a companion paper by Aguirre et al. (2009), hereafter Paper I. This paper presents the catalog of millimeter continuum sources in the surveyed region.

The interpretation of millimeter continuum maps from nearby molecular clouds is guided by preceding studies in an array of molecular gas tracers that developed a detailed understanding of the density structure of clouds (see the review by Evans 1999). The millimeter continuum structures detected in the observations of nearby molecular clouds were molecular cores, i.e., dense structures that are linked to the formation of individual stellar systems. The detection of cores was driven by sensitivity to bright objects with angular scales of tens of arcseconds and filtering out larger angular scale structures.

Although the sensitivity and filtering properties of the instruments remain the same when directed at the Galactic plane, the typical distance to the objects becomes significantly larger $(\sim 5 \mathrm{kpc}$ versus $\sim 250 \mathrm{pc})$. In addition, the range of possible distances spans foreground objects in nearby star-forming regions to bright features viewed from across the Galactic disk. Hence, the physical nature of the objects seen in millimeter surveys of the Galactic plane is less clear than it is for nearby molecular clouds. Guided again by line studies of nearby molecular clouds, surveys such as the BGPS are likely detecting a range of structures within the molecular ISM. The actual objects that are detected are a product of matching the average column density of dust to the angular scales over which the survey is sensitive. Given the distance to the objects, many different types of objects may have an average column density detectable on the $\sim 30^{\prime \prime}$ scales to which the survey is most sensitive.

In addition to the BGPS, other surveys are beginning to explore the Galactic plane in the millimeter dust continuum. The ATLASGAL survey uses the Laboca instrument on the APEX telescope to survey the southern Galactic plane (Schuller et al. 2009) at $\lambda=0.87 \mathrm{~mm}$. After commissioning, the SCUBA2 instrument will be used to survey the plane at $\lambda=0.85 \mathrm{~mm}$ in the JCMT Galactic Plane Survey (JPS; Moore et al. 2005). These 


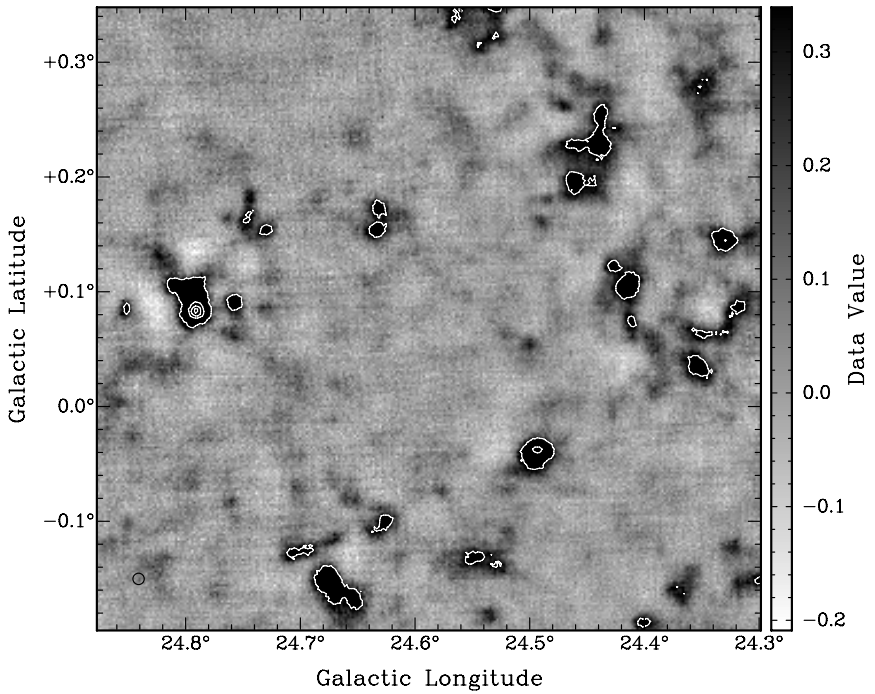

Figure 1. Sample subsection from the BGPS map near $\ell=24^{\circ}$ where the units of the image are $\mathrm{Jy}_{\text {beam }}{ }^{-1}$. White contours indicate the shapes of features above the top end of the gray scale, beginning at $0.33 \mathrm{Jy}$ beam ${ }^{-1}$ and increasing by $0.33 \mathrm{Jy} \mathrm{beam}^{-1}$ linearly. The field highlights several of the structures that informed the design of the cataloging algorithm, including bright compact sources and filamentary structures as well as spatially varying noise and negative bowls around strong sources.

data will complement the open time Hi-GAL project of the Herschel Space Telescope which will observe the inner Galactic plane in several far-infrared bands (Noriega-Crespo \& Molinari 2008). The BGPS and these other surveys are conducted in a blind fashion, meaning that the survey spans all of a large region with no preferential targeting of subregions within that section. Since the survey regions span the low-latitude Galactic plane, they likely cover a large fraction of the star-forming regions in the Galaxy. Hence, the maps will serve as the finding charts for studying dense gas in our Galaxy using the next generation of instrumentation, in particular the Atacama Large Millimeter Array.

This paper presents a catalog of the initial release of the BGPS data suitable for statistical analysis and observational follow-up of the millimeter objects in the Galactic plane. The catalog is produced by a tailor-made algorithm, Bolocat, which was designed and tested in concert with the data reduction and calibration of the BGPS images. Consequently, the algorithm specifically reflects and accounts for the peculiarities in the BGPS images in a fashion that a generic source extraction algorithm could not. We present the catalog through a brief description of the BGPS data (Section 2) and Bolocat (Section 3). We present our validation of the source extraction algorithm in Section 4 and the catalog itself in Section 5. Using observations of the $\mathrm{NH}_{3}$ inversion transitions toward several of the objects, we are able to present constraints on the physical properties of catalog sources which are discussed in Section 6. Finally, we present several summary products from the catalog in Section 7.

\section{THE BOLOCAM GALACTIC PLANE SURVEY}

The BGPS is described in full detail in Paper I. The survey uses the 144-element Bolocam array on the Caltech Submillimeter Observatory, which observes in a band centered at $268 \mathrm{GHz}$ $(1.1 \mathrm{~mm})$ and a width of $46 \mathrm{GHz}$ (Glenn et al. 2003). Of particular note, the bandpass is designed to reject emission from the $\mathrm{CO}(2 \rightarrow 1)$ transition, which is the dominant line contributor at these wavelengths. The survey covers $150 \mathrm{deg}^{2}$ of the Galactic plane, primarily in the first Galactic quadrant. In the first quadrant, the survey ranges from -0.5 to 0.5 in latitude. Survey data are observed in perpendicular scans made in the longitude and latitude direction, with the exception of one block of scans in the W5 region. All of the survey fields are observed in either $1^{\circ} \times 1^{\circ}$ or $3^{\circ} \times 1^{\circ}$ sub-fields which are then jointly reduced producing several individual maps of the plane spanning several degrees.

Imaging of the time stream data requires correcting the signal for the dominant atmospheric contribution in the bolometer array. Since the fundamental assumption in the correction is that the atmosphere is constant across the array, the data are not sensitive to spatial structures with sizes larger than the array (7.5). More detailed analysis shows that structures larger than 3.5 are not fully recovered. An astrophysical image is generated by analyzing the variations of the signal in the time domain and identifying structures due to astrophysical signal in the image domain. By iteratively refining the estimates of instrumental and atmospheric contributions to the time stream, these effects can be decoupled from astrophysical signal producing a map containing only signal and irreducible noise. Of note for the cataloging process, the mapping procedure yields (1) the BGPS map, (2) a model of the signal at each position or equivalently the residual of subtracting the model from the survey map, and (3) a record of the number of times a bolometer sampled a given position on the sky. The pixel scale of the BGPS is set to $7^{\prime \prime} .2$ or 4.58 pixels across the $33^{\prime \prime}$ FWHM beam.

\section{CATALOG METHODOLOGY}

The BGPS catalog algorithm was developed jointly with the mapping process in response to the goals of the BGPS. The BGPS is a large-scale survey of the Galactic plane in a comparatively unexplored waveband. The resulting images contain a wealth of structure as presented in Paper I. A sample image appears in Figure 1 displaying many of the features that motivate the structure of the catalog algorithm. The map contains several bright, compact sources which frequently appear in complexes. The bright sources commonly have low intensity skirts or filaments of fainter emission surrounding or connecting them. In addition, both bright and faint sources have resolved, irregular shapes. The brightest sources commonly have negative bowls around them as a consequence of the principal component analysis (PCA) cleaning during the iterative mapping process (Paper I). These bowls can affect flux density measurements for these objects and depress the significance of surrounding emission. The maps also show noise with a spatially varying rms on scales from pixel (sub-beam) to degree scales. Some artifacts that have not been completely removed by the imaging process still remain, manifesting as correlated noise in the scan directions.

Given these features in the images, we opted to design our own cataloging algorithm rather than adapt a preexisting routine to the BGPS data such as Clumpfind (Williams et al. 1994) or Source EXtractor (Bertin \& Arnouts 1996). Since the physical nature of the objects is not well-constrained, we did not impose prior information on the objects that were to be extracted, such as the functional form of the intensity profile. Instead, the algorithm separates emission into objects defined by a single compact object and nearby emission. The catalog is driven by the need to recover and characterize the properties of the compact sources. The data retained in the catalog needed to be well-suited for follow-up observations, directing observers to the most interesting and bright features in the images. However, filamentary structure commonly appears without an associated 
compact source and we developed a routine that could also characterize isolated filaments of emission as objects. The routine also needed to account for the non-uniform nature of the noise properties over the maps. The algorithm described below is the result of applying these design criteria.

We investigated the behavior of both Clumpfind and Source EXtractor on a subset of the data to verify that a new approach was necessary. Our algorithm shares a common design philosophy with these other approaches. For the recommended user parameters, the Clumpfind algorithm tended to break regions into sub-beam sized objects based on small-scale features in the objects from both real source structure and the spatially varying noise. After exploring the input parameters to Source EXtractor, we were unable to satisfactorily identify filamentary structure in the images. Furthermore, those bright objects that were detected were not divided up into subcomponents. These shortcomings largely arose from (mis)applying these algorithms outside of the domain in which they were originally developed. The unsatisfactory results suggested that developing a tailored cataloging algorithm was necessary.

Sources are extracted from the Bolocam images using a seeded watershed algorithm (Yoo 2004). This algorithm assigns each pixel that is likely to contain emission to a single object based on the structure of the emission and nearby local maxima. Watershed algorithms are so named since they can be used to identify the watershed associated with a given body of water. To make the analogy to the problem at hand, we consider the inverted emission map of a region as a two-dimensional surface. Several initial markers are identified (the seeds) associated with the lowest points of the inverted image (peaks in the original image). The surface is then "flooded" from the starting markers at successively higher levels. At each new level, newly flooded regions are linked to the original seeds associating each point on the surface with one of the starting seeds.

Source identification takes place in a two-step process. First, significant regions of emission are identified based on the amplitude of the signal compared to a local estimate of the noise (Section 3.1). These sources are expanded to include adjacent, low-significance signal (Section 3.2). Second, each region so identified is examined and subdivided further into substructures if there is significant contrast between the sub-objects in the region (Section 3.3).

The algorithm which accomplishes this object identification contains user-determined parameters. In the absence of physical priors, we have set these parameters to reproduce a "by-eye" decomposition of some of the images. We use the same set of parameters to decompose all images in the BGPS and we have validated the parameter selection through in depth testing of the accuracy of the resulting catalog (Section 4). Even though the choice of some parameter values are motivated by the desired behavior of the algorithm, the influence of these choices is well known.

\subsection{Local Noise Estimation}

Objects in the BGPS catalog are identified based on their significance relative to the noise in the map. Owing to variations in the observing conditions and integration time across an individual BGPS field, the underlying noise at each position can vary substantially across a mosaic of several scan blocks. The spatially varying noise $\mathrm{rms}(\sigma)$ is calculated as a function of position using the residual maps from the image production pipeline (Paper I). These maps consist of the actual images less than the source emission model (where the subtraction is

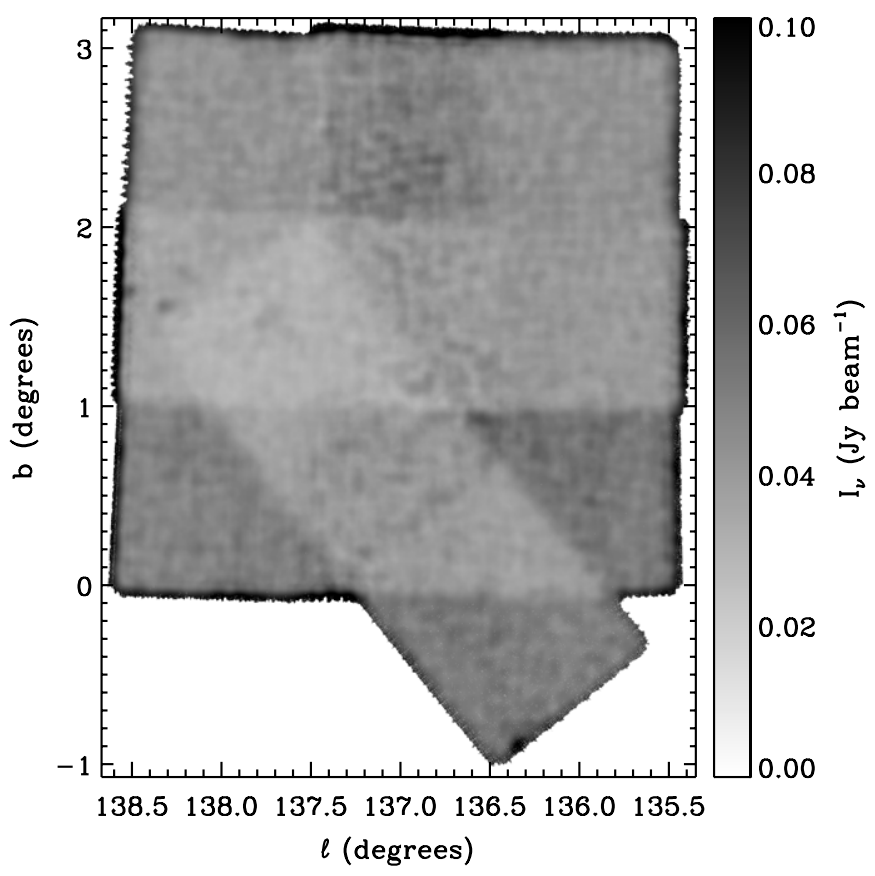

Figure 2. Local variations in the noise for the W5 region of the BGPS. The noise map is generated from the residual map created in the image generation process. The map illustrates variations in the noise level due to variable coverage by the survey and changing observing conditions. The W5 field was selected for display based on its varied survey coverage resulting in the observable structure in the noise map.

performed in the time stream). The residual maps are used in the map reconstruction process and are nominally source-free representations of the noise in the map. We estimate the rms by calculating the median absolute deviation in an $11 \times 11$ pixel box $\left(80^{\prime \prime}\right.$ square) around each position in the map. The size of the box is chosen as a compromise between needing an adequate sample for the estimator and the need to reproduce sharp features in the noise map. The median absolute deviation estimates the rms while being less sensitive to outliers relative to the standard deviation. The resulting map of the rms shows significant pixelto-pixel variation owing to uncertainty in the rms estimator for finite numbers of data. To reduce these fluctuations, we smooth the rms maps with a two-dimensional Savitsky-Golay smoothing filter (Press et al. 1992). The filter is seventh degree and smoothes over a box $12^{\prime}$ on a side. The action of the filter is to reproduce large-scale features in the map such as the boundaries between two noisy regions while smoothing noise regions on beam-size scales. An example map of the rms is shown in Figure 2 after all processing (rms estimate and smoothing).

The statistical noise properties of the map are shown in Figure 3, showing the intensity at each position in the map, $I$ and the signal-to-noise ratio $I / \sigma$. In general, generating an error estimate from the residual map tracks the position-to-position variation of the noise quite well. Transforming the data into significance space (i.e., dividing the data image by the noise estimate) yields a normal distribution with some deviations. As seen in the bottom panel of the figure in particular, there is a wing of high significance outliers associated with the signal in the map. There are more negative outliers than expected, and this arises from pixel-to-pixel variations in the rms based on local conditions. Since our method cannot reproduce these variations perfectly, regions with higher true rms than represented in the rms map will manifest as high significance peaks. There are likely regions for which the rms is an overestimate and such 

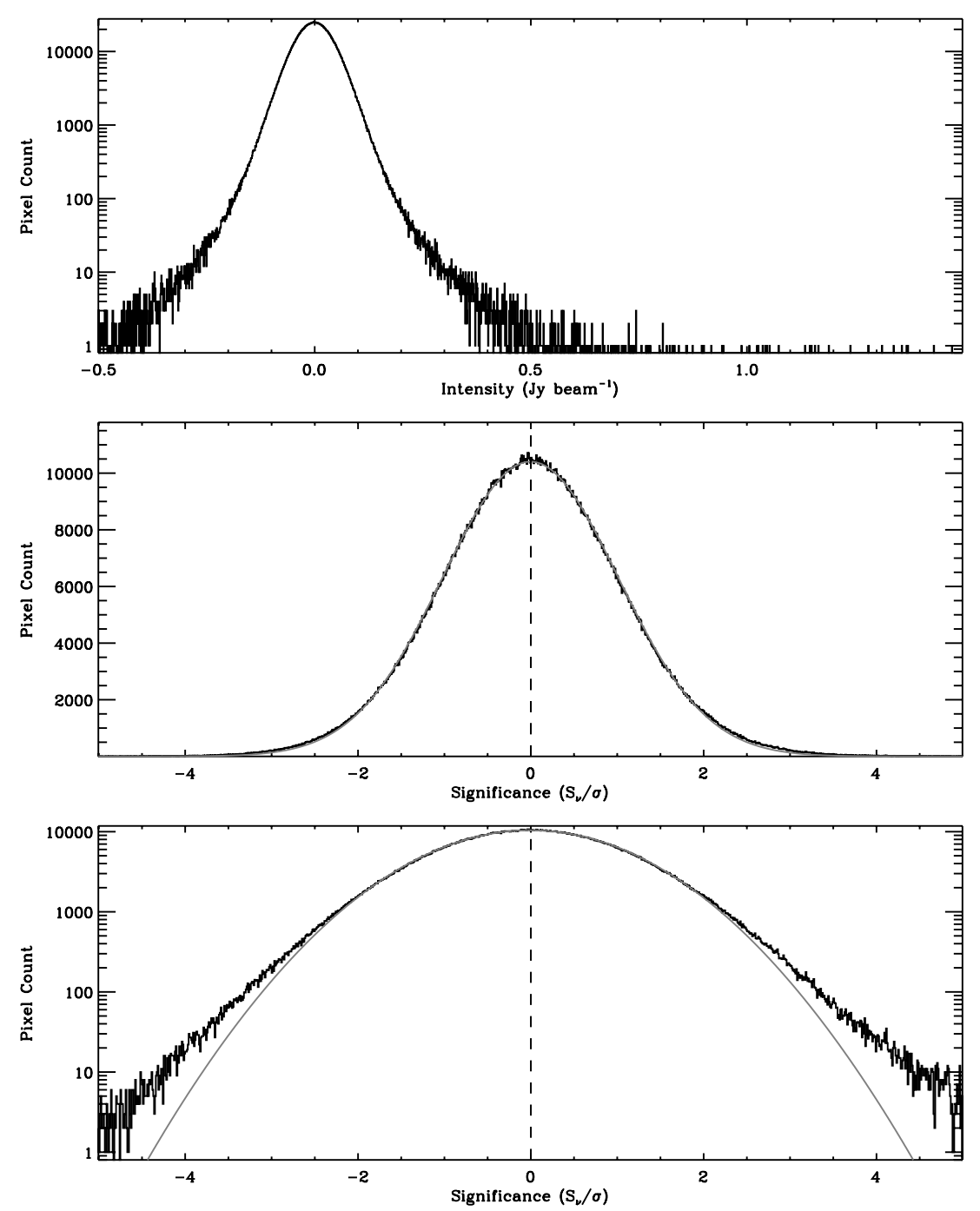

Figure 3. Noise properties for the W5 BGPS field. The top panel shows the histogram of raw data values present in the image, in particular showing a large tail toward high values which arise because of both signal and high noise values at the map edge. The middle panel shows the significance distribution of the image after normalization by the noise estimate derived from the weight map. The bottom panel repeats the data from the middle panel but using a logarithmic scale for the $y$-axis, highlighting the behavior of the distribution at large values of the significance. In both the middle and the bottom panel, a gray curve shows the Gaussian fit to the significance distribution.

regions contribute a slight excess of data near $I / \sigma=0.0$, which is impossible to discern in the face of the large number of data that are expected there. We note that the excess of negative outliers may also result from incomplete removal of astrophysical signal in the model map (see Paper I). In principle, it is possible to track the variations of the noise distribution on sub-beam scales using information in the time domain. We found, however, that the generation of noise statistics from the time stream is unreliable and produces non-normal distributions of significance. However, we do use the time-stream estimate of the noise to identify regions where the residual images are contaminated with astrophysical signal and we replace the rms estimate at positions with spuriously large estimates of the noise with a local average. A Gaussian fit to the distribution of significance values reproduces the shape of the distribution well with the exceptions noted above. Fits to the normal distribution find the central value of the distribution $\langle S\rangle$ zero in all cases $(-0.02 \lesssim\langle I / \sigma\rangle \lesssim 0.02)$. The width of the distribution, which should be identically 1 if $\sigma(\ell, b)$ is a perfect estimate of the noise, is usually within $2 \%$ of 1.00 . Hence, the significance distribution has been transformed to the best approximation of a normal distribution with zero mean and dispersion equal to 1 .

\subsection{Identification of Significant Emission}

Given an estimate of the significance of the image at every position in the data, emission is identified as positive outlying data that are unlikely to be generated by noise. These data form connected regions of positive deviation above a given threshold. We initially mask the data to all regions above $2 \sigma(\ell, b)$. Owing to image artifacts from the Bolocam mapping process, the emission profile shows low-amplitude noise on scales smaller than a beam. As a consequence of this noise, holes in the mask can appear where negative noise fluctuations drive a pixel value below the threshold. Additionally, a set cut at a given threshold tends to produce an artificially jagged source boundary. Finally, the PCA cleaning process creates negative bowls around strong sources. To reduce the influence of these effects, we perform a morphological opening operation on the data with a round structuring element with a diameter of a half a beam-width (see Dougherty 1992, for details). The opening operation minimizes small-scale structure at the edges of the mask on scales less than that of a beam by smoothing the edges of the mask.

Following the opening operation, the remaining regions are expanded to include all connected regions of emission above 

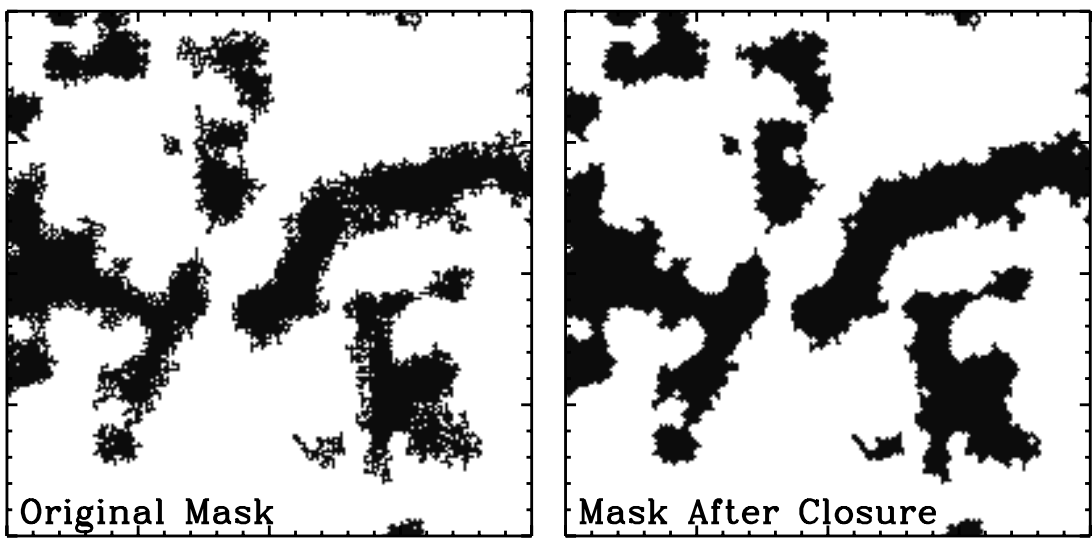

Figure 4. Results of applying a morphological opening and closing operations to a mask. The left-hand panel shows the results of a clip at $2 \sigma$ and the right-hand panel shows the mask after it has been processed by the morphological operations. The opening eliminates artificial structure on scales smaller than that of the beam. The closure smoothes edges and eliminates holes. This largely cosmetic process produces smooth features which enables better source decomposition. The sample region is $24^{\prime}$ on a side.

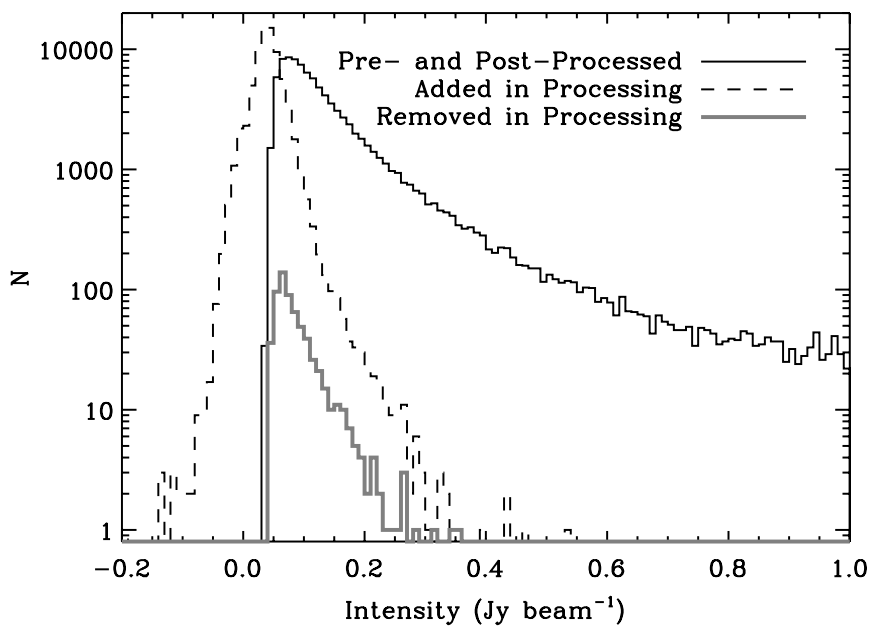

Figure 5. Brightness distribution of pixels included in the emission mask preand post-processing by the opening and closing operators. The added pixels are mostly positive low significance pixels (dashed line) and the removed pixels (gray line) are confined to relatively faint emission. A small fraction (5\%) of the added pixels have negative intensities.

$1 \sigma(\ell, b)$ since regions of marginal significance adjacent to regions of emission are likely to be real. After this rejection, a morphological closing operation is performed with the same structuring element as the previous opening operation. The closing operation smoothes edges and eliminates holes in the mask. This processing minimizes the effects of the sharp cut made in significance on the mask, allowing neighboring, marginal-significance data to be included in the mask. An example of the effects for the opening and closing operations is shown in Figure 4. This results in our final mask containing emission. To assess the effects of the mask processing on our data, we examined the brightness distribution of the pixels in the mask pre- and post-processing by the opening and closing operations. Figure 5 presents the pixel brightness distributions of the regions included in our mask both before and after applying the operators. The eliminated pixels are at low significance and the added pixels include marginal emission as desired. The processed mask also includes some negative intensity pixels in the objects. The added negative pixels are only $5 \%$ by number of the pixels added by mask processing and are not included in the calculation of source properties (Section 3.4).

\subsection{Decomposing Regions of Emission}

The emission mask is a binary array with the value 1 where the map likely contains emission and 0 otherwise. Identification of objects occurs only within the emission mask region. We consider each spatially contiguous region of emission separately and identify substructure within the region. We determine whether this is significant substructure within the map by searching for distinct local maxima within the region. If there is only one distinct local maximum, the entire region is assigned to a single object. Otherwise, the emission is divided among the local maxima using the algorithm described below.

To assess whether a region has multiple distinct local maxima, we search for all local maxima in the region requiring a local maxima to be larger than all pixels within 1 beam FWHM of the candidate. For each pair of local maxima, we then identify the largest contour value that contains both local maxima $\left(I_{\text {crit }}\right)$ and measure the area uniquely associated with each local maximum. If either area is smaller than 3 pixels (as it would be for a noise spike) then the local maximum with the lowest amplitude is removed from consideration (see Figure 6). Furthermore, if either peak is less than $0.5 \sigma$ above $I_{\text {crit }}$ then the maximum with the smaller value of $I_{\nu} / \sigma$ is rejected. This filter dramatically reduces substructure in regions of emission that is due to noise fluctuations. We note that the filter is fairly conservative compared to a by-eye decomposition. As a consequence, compound objects are de-blended less aggressively than a manual decomposition. The rejection criteria are evaluated for every pair of local maxima in a connected region, so every local maximum that remains after decimation is distinct from every other remaining maximum.

The final set of local maxima are thus well separated from their neighbors, have significant contrast with respect to the larger complex, and have a significant portion of the image associated with them. Using this set of local maxima, we partition the regions of emission into catalog objects. This partitioning is accomplished using a seeded watershed algorithm. Such an algorithm is, in principle, similar to the methods used in Clumpfind (Williams et al. 1994) in molecular line astronomy or Source EXtractor (Bertin \& Arnouts 1996) in optical astronomy. To effect the decomposition, the emission is contoured with a large number of levels (500 in this case). For each level beginning with the highest, the algorithm clips the emission at that level and examines all the resulting isolated sub-regions. 


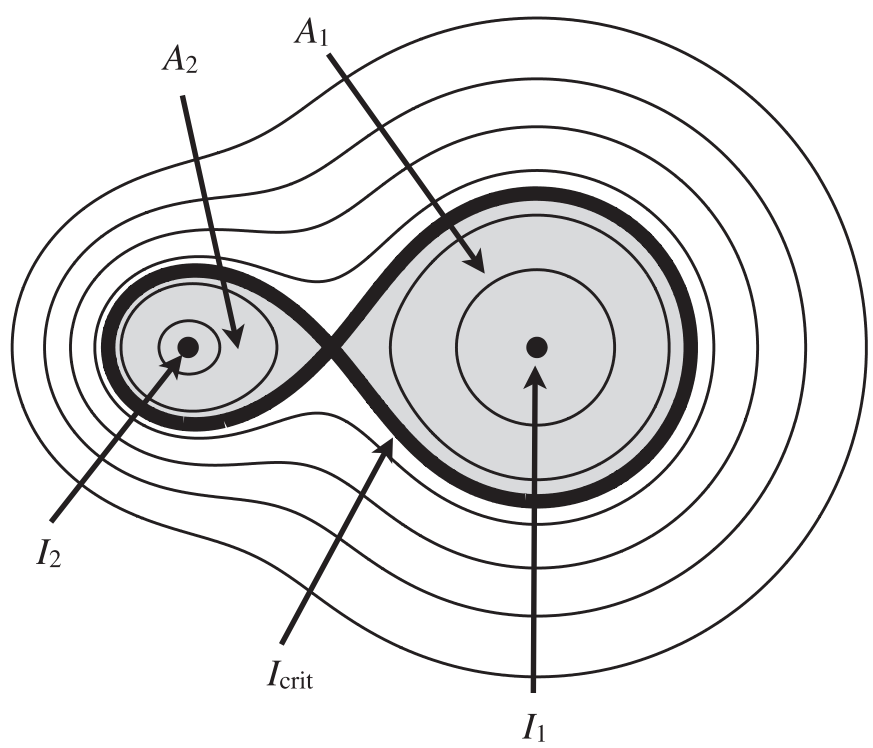

Figure 6. Schematic contour diagram of elimination criterion for local maxima. Each of a pair of local maximum (here $I_{1}, I_{2}$ ) has an area uniquely associated with that local maximum (i.e., above a critical contour $I_{\text {crit }}$ ). If the difference between $I_{i} / \sigma$ and $I_{\text {crit }} / \sigma$ is smaller than 0.5 then the smaller amplitude local maximum is removed from the list of candidate local maxima. If the smaller area uniquely associated with a maximum (here $A_{2}$ ) is smaller than 3 pixels, then that maximum is removed.

For any pixels not considered at a higher level, the algorithm assigns those pixels to the local maximum to which they are connected by the shortest path contained entirely within the subregion. If there is only one local maximum within the subregion, the assignment of the newly examined pixels is trivial. The test contour levels are marched progressively lower until all the pixels in the region are assigned to a local maximum. An example of the partitioning of a region is shown in Figure 7.

\subsection{Measuring Source Properties}

A BGPS catalog object is defined by the assignment algorithm established above which yields a set of pixels with known Galactic coordinates $\left(\ell_{j}, b_{j}\right)$ and associated intensities $I_{j}\left(\mathrm{Jy} \mathrm{beam}^{-1}\right)$. Source properties are determined by emission-weighted moments over the coordinate axes of the BGPS image. Positions in the mask with negative intensity are not included in the calculation of properties. For example, the centroid Galactic longitude of an object is

$$
\ell_{\mathrm{cen}}=\frac{\sum_{j} \ell_{j} I_{j}}{\sum_{j} I_{j}}
$$

with $b_{\text {cen }}$ defined in an analogous fashion. The sizes of the objects are determined from the second moments of the emission along the coordinate axes:

$$
\begin{gathered}
\sigma_{\ell}^{2}=\frac{\sum_{j}\left(\ell_{j}-\ell_{\mathrm{cen}}\right)^{2} I_{j}}{\sum_{j} I_{j}} \\
\sigma_{b}^{2}=\frac{\sum_{j}\left(b_{j}-b_{\mathrm{cen}}\right)^{2} I_{j}}{\sum_{j} I_{j}} \\
\sigma_{\ell b}=\frac{\sum_{j}\left(\ell_{j}-\ell_{\mathrm{cen}}\right)\left(b_{j}-b_{\mathrm{cen}}\right) I_{j}}{\sum_{j} I_{j}} .
\end{gathered}
$$

The principal axes of the flux density distribution are determined by diagonalizing the tensor

$$
\mathbf{I}=\left[\begin{array}{cc}
\sigma_{\ell}^{2} & \sigma_{\ell b} \\
\sigma_{\ell b} & \sigma_{b}^{2}
\end{array}\right]
$$

The diagonalization yields the major $\left(\sigma_{\mathrm{maj}}\right)$ and minor $\left(\sigma_{\mathrm{min}}\right)$ axis dispersions as well as the position angle of the source. Since the moments are calculated for an emission mask that has been clipped at a positive significance level, these estimates will slightly underestimate the sizes of the sources leading to size estimates that could be smaller than the beam size. Once projected into the principal axes of the intensity distribution, the angular radius of the object can be calculated as the geometric mean of the deconvolved major and minor axes:

$$
\theta_{R}=\eta\left[\left(\sigma_{\text {maj }}^{2}-\sigma_{\mathrm{bm}}^{2}\right)\left(\sigma_{\min }^{2}-\sigma_{\mathrm{bm}}^{2}\right)\right]^{1 / 4} .
$$

Here $\sigma_{\mathrm{bm}}$ is the rms size of the beam $\left(=\theta_{\mathrm{FWHM}} / \sqrt{8 \ln 2}=14^{\prime \prime}\right)$ and $\eta$ is a factor that relates the rms size of the emission distribution to the angular radius of the object determined. The appropriate value of $\eta$ to be used depends on the true emission distribution of the object and its size relative to the beam. We
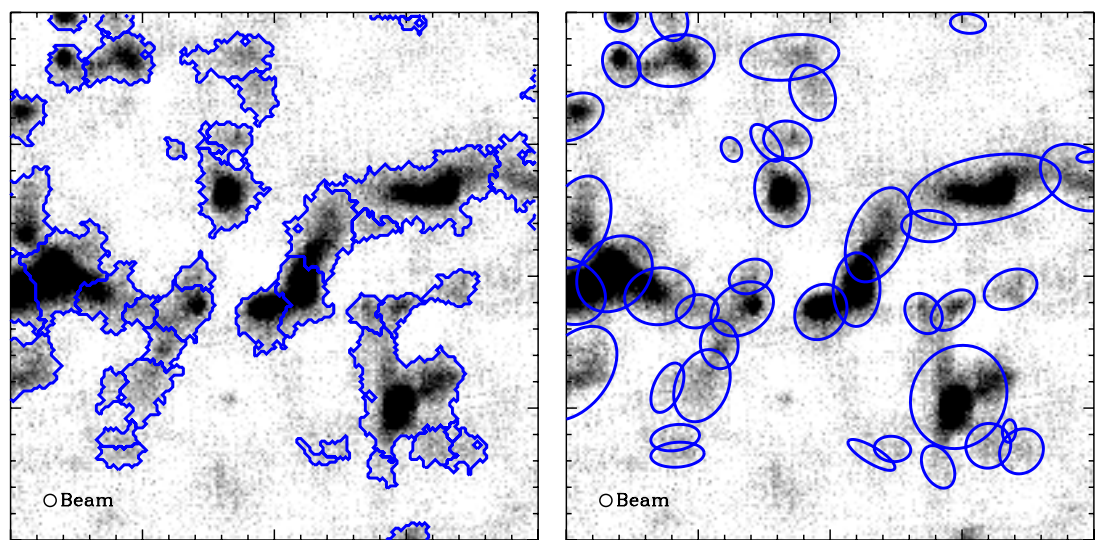

Figure 7. Example of the watershed algorithm applied to the same region shown in Figure 4 (left) and the measured source shapes (right). The gray-scale image shows the actual BGPS map image which runs on a linear scale from 0 to $0.2 \mathrm{Jy} \mathrm{beam}^{-1}$. In the left panel, the blue boundaries indicate the regions identified using the seeded watershed algorithm. In the right panel, the ellipses indicate the shapes derived from the moment methods applied to the extracted objects. The example region shown is from the original $\ell=32^{\circ}$ BGPS maps and is $24^{\prime}$ on a side.

(A color version of this figure is available in the online journal.) 
have elected to use a value of $\eta=2.4$, which is the median value derived for a range of models consisting of a spherical, emissivity distribution $\mathrm{j}(r) \propto r^{\gamma}$ with $-2<\gamma<0$, a range of radii relative to the beam $0.1<\theta_{R} / \theta_{\mathrm{FWHM}}<3$ at a range of significance (peak signal to noise ranging from 3 to 100). The range of $\eta$ for the simulations is large, varying by more than a factor of 2. If a specific source model is required for a follow-up study, the appropriate value of $\eta$ should be calculated for this model and the catalog values can be rescaled to match.

Uncertainties in the derived quantities are calculated by propagating the pixel-wise estimates of the flux density uncertainties through the definitions of the quantities. The coordinate axes are assumed to have negligible errors. The moment descriptions of position, size, and orientation are exact for Gaussian emission profiles and generate good Gaussian approximations for more complex profiles. An example of the derived shapes of the catalog objects is shown in Figure 7 for comparison with the shapes of the regions from which they are derived. In comparing the images, it is clear that the irregularly shaped catalog objects can produce elliptical sources that vary from what a Gaussian fit would produce. However, in most cases the moment approximations characterize the object shapes well.

Since the extracted objects are often asymmetric, the centroid of the emission does not correspond to the peak of emission in an object. Because of this possible offset, we also report the coordinates of the maximum of emission. To minimize the effect of noise on a sub-beam scale, we replace the map values with the median value in a $5 \times 5$ pixel $\left(36^{\prime \prime}\right)$ box around the position and determine the position of the maximum value in the smoothed map. While this reduces the positional accuracy of the derived maximum, it dramatically improves the stability of the estimate. Since the peaks of emission are rarely point sources, the loss of resolution is made up for by the increased signal to noise in the map. These coordinates are reported as $\ell_{\max }$ and $b_{\max }$. If the catalog is being used to follow-up on bright millimeter sources in the plane these coordinates are preferred over the centroid coordinates. Moreover, since the maxima are less subject to decomposition ambiguities, we name our sources

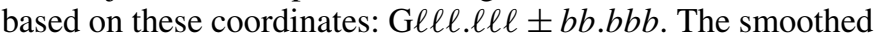
map is only used for the calculation of the position for the maximum; all other properties are calculated from the original BGPS map.

The flux densities of the extracted objects are determined by measuring the flux density in an aperture and by integrating the flux density associated with the region defined by each object in the watershed extraction. In the catalog, three flux density values for each source are reported, corresponding to summing the intensity in apertures with diameters $d_{\text {app }}=40$, " 80", and $120^{\prime \prime}$. We refer to these flux densities as $S_{40}, S_{80}$, and $S_{120}$, respectively. Because many of the objects are identified in crowded fields, we refrain from a general "sky" correction since inspection of the noise statistics suggests that the average value of the sky is quite close to zero. One notable exception to this case is where there are pronounced negative bowls in the emission around the object. Because large apertures may contain parts of these negative bowls, the aperture flux densities of bright objects are likely underestimated. In addition, we also report the object flux density value $(S)$, which is determined by integrating the flux density from all pixels contributing to the object in the watershed decomposition of the emission:

$$
S \equiv \sum_{i} I_{i} \Delta \Omega_{i}
$$

where $\Delta \Omega_{i}$ is the angular size of a pixel. The object flux density cannot, by definition of the regions, contain negative bowls, but the bowls indicate that emission is not fully restored to these values, which are thus likely underestimates of the true flux density for bright objects. Since objects typically have angular radii $\sim 60^{\prime \prime}, 120^{\prime \prime}$ apertures do not contain all the flux density. Hence, $S \gtrsim S_{120}>S_{80}>S_{40}$. Since the surface brightness at each position in the map has an associated uncertainty (Section 3.1), we calculate the uncertainty in the flux density measurements by summing the uncertainties in quadrature. We further account for the uncertainty in the size of the beam owing to the uncertainties of the bolometer positions in the focal plane of the instrument (see Paper I). The latter effect produces a fractional uncertainty in the beam area of $\delta \Omega_{\mathrm{bm}} / \Omega_{\mathrm{bm}}=0.06$. Thus, the uncertainty in a flux density measurement (either aperture or integrated) is

$$
\delta S \equiv \sqrt{\frac{\Omega_{i}}{\Omega_{\mathrm{bm}}} \sum_{i} \sigma_{i}^{2}+\left(\frac{\delta \Omega_{\mathrm{bm}}}{\Omega_{\mathrm{bm}}} S\right)^{2}},
$$

where the ratio $\Omega_{i} / \Omega_{\mathrm{bm}}$ accounts for the multiple pixels per independent resolution element. The flux density errors do not account for any systematic uncertainty in the calibration of the image data which is estimated to be less than $50 \%$ (Paper I). We discuss the flux densities in more detail in Section 7.2 below.

\section{SOURCE RECOVERY EXPERIMENTS}

In order to assess the properties of catalog sources compared to the actual distribution of emission on the sky, we have created simulated maps of the emission, inserting sources with known properties. The generation of these simulated images relies on the actual observed data for the $\ell=111^{\circ}$ region of the survey. This field was selected for analysis based on its uniform noise properties and well-recovered astrophysical signal. Most of the astrophysical signal is identified in the time series data from the instrument and removed, leaving only irreducible (i.e., shot) noise in the time series with small contributions due to atmospheric, instrumental (e.g., the pickup of vibrations), and astrophysical signals (see Paper I for more detail). Then a series of Gaussian objects was sampled into the data time stream. The simulation time streams are then processed in a fashion identical to the final BGPS survey images resulting in a suite of test images to test the catalog algorithm on. Gaussian brightness profiles do not adequately represent all the structure seen in the BGPS maps, but they do capture the essential features of compact sources. The complicated interplay of extended structure with the mapping process likely affects the validity of the following tests. However, the behavior for point-like or small sources should be well reproduced. Finally, some of the astrophysical signal remains in the residual image which affects some of our tests to a small degree.

\subsection{Completeness}

The primary test of the algorithm is the degree of completeness of the catalog at various flux density limits. To this end, we have inserted a set of point sources into the time stream with flux densities uniformly distributed between $0.01 \mathrm{Jy}$ and $0.2 \mathrm{Jy}$ arranged in a grid. We then process the simulated data with the source extraction algorithm and compare the input and output source catalog. The results of the completeness study are shown in Figure 8. For this field, the mean noise level is 


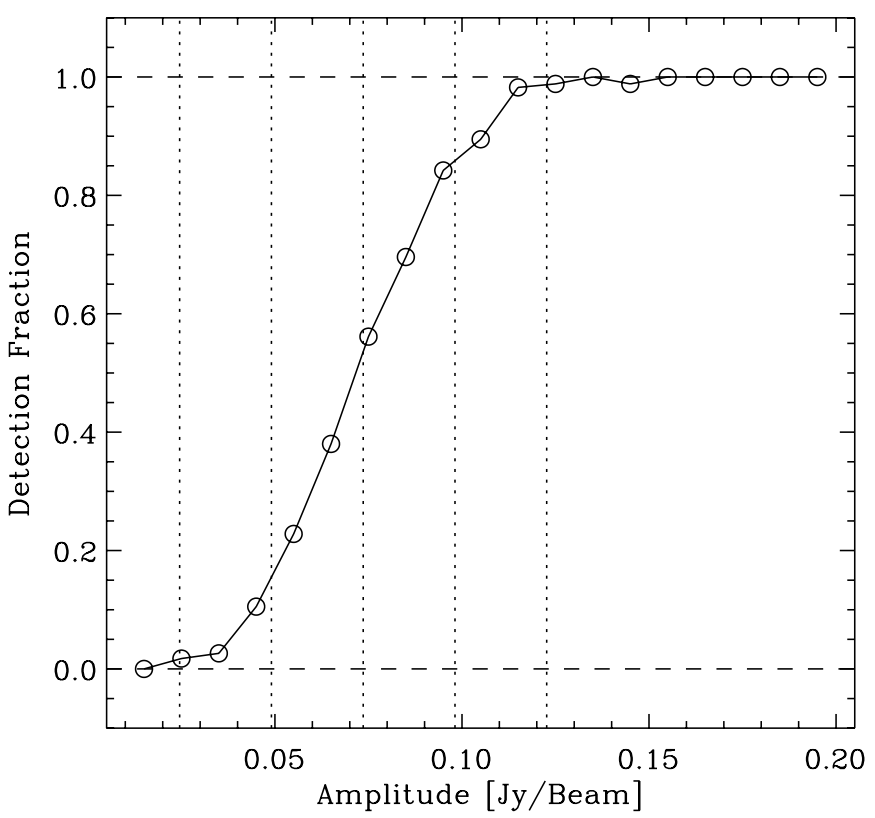

Figure 8. Completeness fraction derived from fake source tests in a survey field The fraction of sources recovered is plotted as a function of input source flux density. The vertical dotted lines indicate $\{1,2,3,4,5\} \sigma$. The completeness tests indicate that the catalog is complete at the $>99 \%$ limit for source flux densities $\geqslant 5 \sigma$.

$\sigma=24$ mJy beam $^{-1}$. The catalog is $>99 \%$ complete at the $5 \sigma$ level. Below this level, the completeness fraction rolls off until no sources are detected at $2 \sigma$. For this regular distribution of sources, there are no false detections throughout the catalog (even at the $2 \sigma$ level). The catalog parameters described above are selected to minimize the number of false detections. Even though the thresholding of the map is performed at the relatively low $2.0 \sigma$ level, the wealth of restrictions placed on the extracted regions combine to raise the effective completeness limit to $5 \sigma$. We note that the grid arrangement of test sources means that confusion effects are not accounted for in the flux limit.

In Figure 9, we present the estimated completeness limit as a function of Galactic longitude for the BGPS. The $\ell=111^{\circ}$ region has a relatively low value for the noise rms with several fields being up to a factor of 4 larger than the completeness test field. We assume that the completeness limit scales with the local rms. The assumption is motivated since all catalog source extraction and decomposition is performed in the significance map rather than in the image units. Thus, the absolute value of the image data should not matter. Over $>98 \%$ of the cataloged area, the completeness limit is everywhere less than $0.4 \mathrm{Jy}$, thus the survey, as a whole can be taken to be $98 \%$ complete at the 0.4 Jy level.

\subsection{Decomposition Fidelity}

In addition to completeness limits, we investigated the fidelity of the algorithm by comparing extracted source properties to the input source distributions. We compared the extracted flux density values to the input flux density values as shown in Figure 10. These simulations compare input and recovered properties for resolved sources with intrinsic FWHMs equal to 2.4 times that of the beam. The flux densities of the sources are varied over a significant range, and we measure the recovered values for the flux density using the $40^{\prime \prime}, 80^{\prime \prime}$, and $120^{\prime \prime}$ apertures and we also compute the integrated flux density in the object. Since the small apertures only contain a fraction of the total flux density, their average flux density recovery is naturally smaller than the input. As the aperture becomes better matched to the source size, the flux density recovery improves, in agreement with analytic expectations based on the source profile. Since many objects are larger than the $40^{\prime \prime}$ and $80^{\prime \prime}$ apertures, catalog flux density values can be viewed in terms of surface brightnesses within these apertures.

We completed an additional suite of simulations, including sources with sizes significantly larger than the beam. These simulations are used in Paper I to test the effect of the deconvolution process, but we also examine the results of the source extraction. In Figure 10, we show the recovered source size as a function of input size. In general, smaller sources are better recovered with objects much larger than the beam having a smaller size found. Radii of objects become biased for sources with sizes $\gtrsim 200^{\prime \prime}$, consistent with the experiments on deconvolution discussed in Paper I. Because of the image processing methods used, objects with sizes larger than this should be treated with caution. However, we note that no sources in the actual catalog are found with sizes $>220^{\prime \prime}$ and the mean size is only $60^{\prime \prime}$ so objects of these sizes and their properties are likely to be recovered. The catalog algorithm thus does not introduce any additional bias into the properties of the recovered sources. However, the sizes of objects may not be completely recovered in the mapping process and thus the extracted properties may not accurately reflect the true properties of sources because of limitations in the map making process. These map properties should be viewed with the caveats presented in Paper I.

\subsection{Deblending}

A primary question of the catalog algorithm is how well it separates individual sources into their appropriate subcomponents. Any approach to source extraction and decomposition will be unable to separate some source pairs accurately. However, the limiting separation for source extraction is determined by the method. Our approach is further complicated by the assignment of emission from every position in the catalog to at most one object. Thus, blended sources are difficult to extract separately unless the individual local maxima that define the sources are apparent according to the criteria in Section 3.3. To assess how the catalog algorithm recovers sources, we generated maps of sources with a random distribution in position and processed the maps with the catalog algorithm. The input sources have elliptical Gaussian profiles with a uniform distribution of major and minor axis sizes between our beam size (33") and $72^{\prime \prime}$, which represent typical objects seen in the BGPS maps. The input sources are oriented randomly. For each pair of input sources, we determine whether the pair is assigned to the same catalog object. We then measure the fraction of blended sources as a function of separation. The results of the experiment are shown in Figure 11 which indicates that the algorithm resolves most sources that are separated by $>75^{\prime \prime}$ or 2 beam FWHM. For point sources, the nominal resolution limit is $\approx 1$ beam FWHM; however the algorithm requires $\sim 2$ FWHM between local maxima so this limit on deblending is consistent with the design. Furthermore, the test objects are resolved, which complicates the analysis. Thus, Figure 11 also indicates the fraction of blended sources for pairs of the smallest sources and for pairs of the largest sources (i.e., both sources are in the bottom $15 \%$ of the size distribution or the top $15 \%$, respectively). In separating the data based on size, we can assess the degree to which source structure will complicate deblending. As expected, pairs of small sources are easier to resolve $(50 \%$ are separated for distances of 


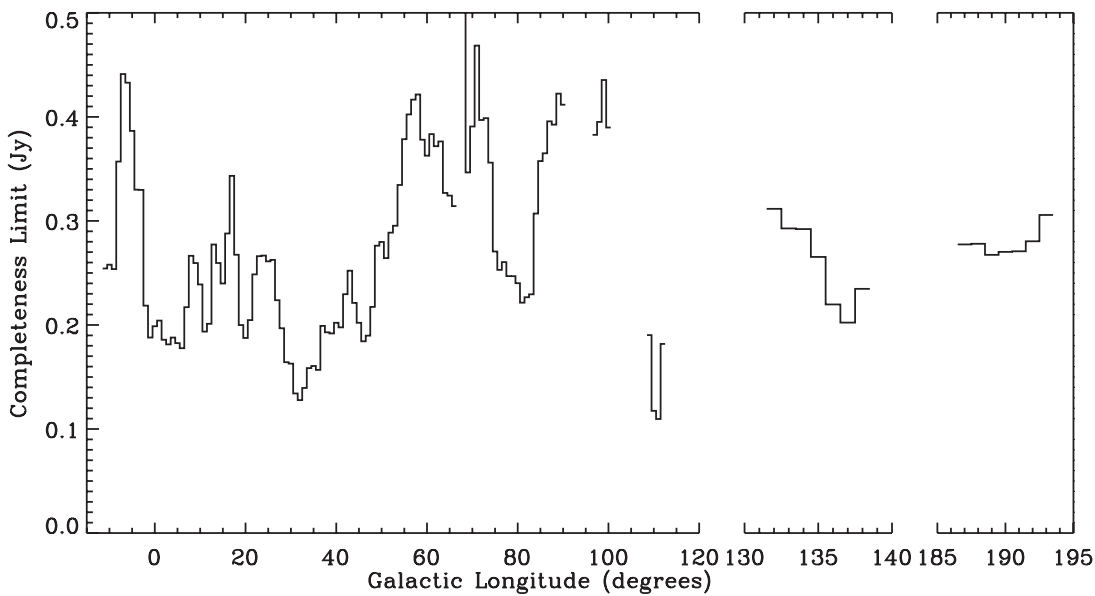

Figure 9. 99\% completeness limit in flux density for point sources plotted as a function of Galactic longitude. The completeness limit is 5 times the median rms value in $1^{\circ}$ bins across the survey. The significant variation in the completeness limit stems from the variable observing conditions and integration time for each field in the survey.
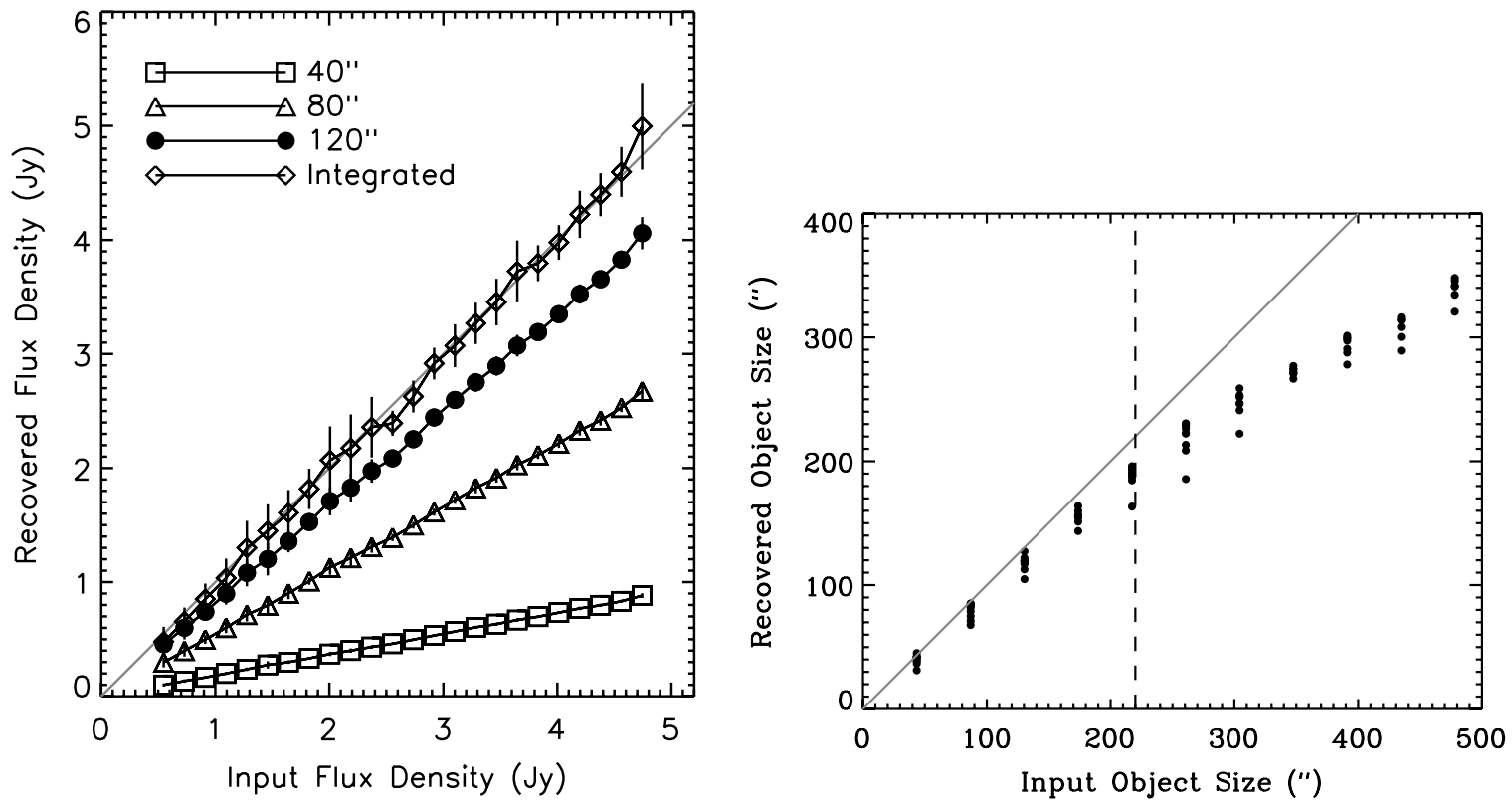

Figure 10. Recovery of source flux density and source size for simulated observations. The left-hand panel compares input and recovered source flux densities for resolved objects $\left(\mathrm{FWHM}=2.4 \theta_{\text {beam }}=79^{\prime \prime}\right)$. The right-hand panel shows the size measured in the simulated objects for an array of input object sizes. The vertical dashed line at $210^{\prime \prime}$ indicates the largest major-axis size of objects actually found in the BGPS implying source sizes are typically well recovered.

$\left.60^{\prime \prime}\right)$ and pairs of large sources more difficult to deblend (50\% at $\left.85^{\prime \prime}\right)$. The large source curve crosses the population curve at large separations $\left(120^{\prime \prime}\right)$ because of small number statistics rather than any algorithmic feature.

\section{THE CATALOG}

The BGPS catalog contains 8358 sources extracted using the algorithm described in Section 3. An excerpt from the catalog is shown in Table 1. The catalog data presented here are derived from the original BGPS image data released in 2009 June (image version 1.0). The full and up-to-date catalog and the BGPS image data are hosted at the Infrared Processing and Analysis Center ${ }^{11}$, which will reflect incremental improvements in image processing, calibration, and cataloging.

\footnotetext{
11 http://irsa.ipac.caltech.edu/data/BOLOCAM_GPS/
}

\section{THE PHYSICAL PROPERTIES OF CATALOG SOURCES}

The BGPS data contain a wealth of sources extracted from the images with a wide variety of source properties. The physical meaning of a catalog source remains unclear, however. Ascribing nomenclature such as a "clump" or a "core" to catalog objects may be misleading in certain cases as these terms can refer to specific density, mass and size regimes (e.g., Williams et al. 2000). In this section, we explore the range of parameter space a BGPS catalog object may occupy. We also refer readers to two additional discussions of BGPS sources in the Galactic center (Bally et al. 2009) and in the Gem OB1 star-forming region in the outer Galaxy (Dunham et al. 2010). These two studies consider the source properties in regions where most of the sources are likely found at a known distance. In contrast, blind observations of the Galactic plane can span a range of distances.

To facilitate understanding BGPS source properties, we include Robert F. Byrd Green Bank Telescope (GBT) observations 


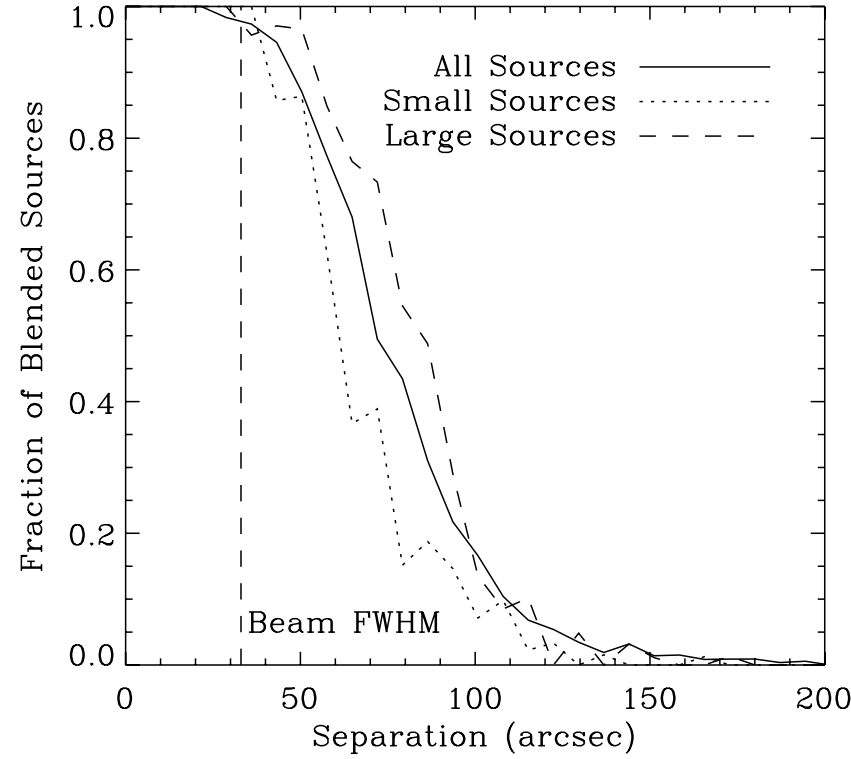

Figure 11. Fraction of source pairs that remain blended as a function of source pair separation. The solid line shows the blending fraction for all source pairs. Since the sources are elliptical Gaussians, the dashed and dotted curves show the same function plotted only for the source pairs with mean sizes in the top and bottom $15 \%$, indicating that large resolved sources are more difficult to separate algorithmically.

of the $\mathrm{NH}_{3}(1,1)$ and $(2,2)$ inversion transitions toward a set of BGPS detections in the $\ell \sim 31^{\circ}$ field. In making this comparison, we assume that the ammonia emission comes from the same regions as are responsible for the millimeter continuum emission, an assumption supported by studies in local molecular clouds (e.g., Rosolowsky et al. 2008; Friesen et al. 2009). These data are presented formally and in much greater depth elsewhere (Dunham et al. 2010). Here we note that the ammonia data provide complementary information on the line-ofsight velocities $\left(V_{\mathrm{LSR}}\right)$, line widths $\sigma_{V}$, and kinetic temperatures of dense gas $T_{K}$. The velocity information, in particular, yields distance estimates to the sources by assuming that the radial motion of the line emitters arises entirely from their orbital motion around the Galactic center. We then use the kinematic model of Reid et al. (2009) to estimate the distances to BGPS objects. The Reid et al. model uses VLBI parallaxes to establish the distances to several high-mass star-forming regions. One of the primary results of their work is a differentiation between the kinematic properties of high-mass star-forming regions and other components of the Galactic disk. Since the BGPS sources are likely associated with (high-mass) star-forming regions, their refined model is appropriate for our work.

The ammonia observations were taken before the BGPS catalog was complete, and the GBT targets were selected byeye from an early version of the BGPS images. Hence, there is not a one-to-one correspondence between BGPS catalog sources and ammonia observations. We match each ammonia spectrum to the BGPS catalog source for which the pointing center for the GBT observation falls into the region assigned to the BGPS source. If multiple GBT observations fall within a BGPS source, we consider only the spectrum with the highest integrated intensity in the $(1,1)$ transition. In this fashion, we match 42 ammonia spectra with BGPS sources. We estimate

Table 1

The BGPS Catalog (Excerpt) ${ }^{\mathrm{a}}$

\begin{tabular}{|c|c|c|c|c|c|c|c|c|c|c|c|c|c|}
\hline (1) & Name & $\begin{array}{c}\ell_{\max } \\
\left(^{\circ}\right) \\
(3)\end{array}$ & $\begin{array}{c}b_{\max } \\
\left({ }^{\circ}\right) \\
(4)\end{array}$ & $\begin{array}{c}\ell \\
\left({ }^{\circ}\right) \\
(5) \\
\end{array}$ & $\begin{array}{c}b \\
\left({ }^{\circ}\right) \\
(6)\end{array}$ & $\begin{array}{c}\sigma_{\text {maj }} \\
\left({ }^{\prime \prime}\right) \\
(7)\end{array}$ & $\begin{array}{c}\sigma_{\min } \\
\left({ }^{\prime \prime}\right) \\
(8)\end{array}$ & $\begin{array}{c}\text { P.A. } \\
\left({ }^{\circ}\right) \\
(9)\end{array}$ & $\begin{array}{c}\theta_{R} \\
\left({ }^{\prime \prime}\right) \\
(10)\end{array}$ & $\begin{array}{l}S_{40} \\
(\mathrm{Jy}) \\
(11)\end{array}$ & $\begin{array}{l}S_{80} \\
(\mathrm{Jy}) \\
(12)\end{array}$ & $\begin{array}{c}S_{120} \\
(\mathrm{Jy}) \\
(13)\end{array}$ & $\begin{array}{c}S \\
(\mathrm{Jy}) \\
(14)\end{array}$ \\
\hline 1 & $\mathrm{G} 000.000+00.057$ & 0.000 & 0.057 & 0.004 & 0.063 & 32 & 17 & 89 & 38 & $0.19 \pm 0.06$ & $0.56 \pm 0.11$ & $1.01 \pm 0.18$ & $0.67 \pm 0.12$ \\
\hline 2 & $\mathrm{G} 000.004+00.277$ & 0.004 & 0.277 & 0.007 & 0.276 & 39 & 34 & 145 & 80 & $0.21 \pm 0.04$ & $0.59 \pm 0.09$ & $0.99 \pm 0.13$ & $1.40 \pm 0.17$ \\
\hline 3 & G000.006 - 00.135 & 0.006 & -0.135 & 0.019 & -0.138 & 43 & 32 & 115 & 82 & $0.22 \pm 0.05$ & $0.71 \pm 0.11$ & $1.39 \pm 0.17$ & $1.41 \pm 0.19$ \\
\hline 4 & G000.010 + 00.157 & 0.010 & 0.157 & 0.019 & 0.156 & 62 & 24 & 80 & 81 & $0.61 \pm 0.06$ & $1.62 \pm 0.15$ & $2.33 \pm 0.21$ & $3.55 \pm 0.30$ \\
\hline 5 & G000.016 - 00.017 & 0.016 & -0.017 & 0.012 & -0.014 & 50 & 35 & 44 & 93 & $1.24 \pm 0.10$ & $3.68 \pm 0.26$ & $6.23 \pm 0.43$ & $10.94 \pm 0.74$ \\
\hline 6 & G000.018 - 00.431 & 0.018 & -0.431 & 0.016 & -0.431 & 23 & 8 & 97 & $\ldots$ & $0.08 \pm 0.05$ & $0.16 \pm 0.09$ & $0.19 \pm 0.14$ & $0.15 \pm 0.07$ \\
\hline 7 & G000.020 + 00.033 & 0.020 & 0.033 & 0.019 & 0.036 & 38 & 32 & 54 & 77 & $0.83 \pm 0.09$ & $2.68 \pm 0.22$ & $4.86 \pm 0.37$ & $6.07 \pm 0.46$ \\
\hline 8 & G000.020 - 00.051 & 0.020 & -0.051 & 0.021 & -0.052 & 34 & 26 & 85 & 62 & $1.14 \pm 0.10$ & $3.43 \pm 0.25$ & $5.75 \pm 0.41$ & $6.37 \pm 0.46$ \\
\hline 9 & $\mathrm{G} 000.022+00.251$ & 0.022 & 0.251 & 0.025 & 0.250 & 26 & 14 & 90 & $\ldots$ & $0.10 \pm 0.04$ & $0.24 \pm 0.09$ & $0.43 \pm 0.13$ & $0.26 \pm 0.08$ \\
\hline 10 & G000.034 - 00.437 & 0.034 & -0.437 & 0.037 & -0.438 & 17 & 11 & 92 & $\ldots$ & $0.06 \pm 0.05$ & $0.14 \pm 0.09$ & $0.18 \pm 0.14$ & $0.11 \pm 0.06$ \\
\hline 11 & G000.042+00.205 & 0.042 & 0.205 & 0.041 & 0.208 & 44 & 24 & 155 & 70 & $0.36 \pm 0.05$ & $1.21 \pm 0.12$ & $2.15 \pm 0.19$ & $2.61 \pm 0.23$ \\
\hline 12 & G000.044 - 00.133 & 0.044 & -0.133 & 0.049 & -0.135 & 29 & 20 & 97 & 45 & $0.14 \pm 0.05$ & $0.42 \pm 0.10$ & $0.76 \pm 0.16$ & $0.62 \pm 0.12$ \\
\hline 13 & G000.044 - 00.285 & 0.044 & -0.285 & 0.051 & -0.282 & 26 & 10 & 137 & $\ldots$ & $0.08 \pm 0.04$ & $0.33 \pm 0.08$ & $0.60 \pm 0.13$ & $0.15 \pm 0.06$ \\
\hline 14 & G000.046 + 00.111 & 0.046 & 0.111 & 0.049 & 0.108 & 39 & 24 & 142 & 64 & $0.33 \pm 0.05$ & $0.92 \pm 0.11$ & $1.40 \pm 0.16$ & $1.83 \pm 0.20$ \\
\hline 15 & G000.046 - 00.041 & 0.046 & -0.041 & 0.047 & -0.037 & 34 & 29 & 168 & 67 & $0.47 \pm 0.07$ & $1.67 \pm 0.17$ & $3.36 \pm 0.28$ & $3.21 \pm 0.28$ \\
\hline 16 & G000.046 - 00.297 & 0.046 & -0.297 & 0.043 & -0.294 & 30 & 21 & 141 & 49 & $0.11 \pm 0.04$ & $0.32 \pm 0.08$ & $0.52 \pm 0.12$ & $0.48 \pm 0.10$ \\
\hline 17 & $\mathrm{G} 000.048+00.065$ & 0.048 & 0.065 & 0.047 & 0.070 & 13 & 12 & 127 & $\ldots$ & $0.14 \pm 0.05$ & $0.56 \pm 0.11$ & $1.34 \pm 0.17$ & $0.19 \pm 0.06$ \\
\hline 18 & $\mathrm{G} 000.052+00.027$ & 0.052 & 0.027 & 0.048 & 0.029 & 39 & 33 & 161 & 79 & $1.30 \pm 0.11$ & $3.78 \pm 0.27$ & $6.05 \pm 0.43$ & $8.10 \pm 0.56$ \\
\hline 19 & G000.052 - 00.159 & 0.052 & -0.159 & 0.051 & -0.159 & 21 & 19 & 48 & 33 & $0.11 \pm 0.05$ & $0.33 \pm 0.09$ & $0.67 \pm 0.14$ & $0.33 \pm 0.09$ \\
\hline 20 & G000.054 - 00.209 & 0.054 & -0.209 & 0.055 & -0.207 & 37 & 36 & 73 & 80 & $0.87 \pm 0.07$ & $2.56 \pm 0.18$ & $4.09 \pm 0.29$ & $5.76 \pm 0.41$ \\
\hline 21 & G000.066 + 00.209 & 0.066 & 0.209 & 0.067 & 0.211 & 34 & 28 & 115 & 66 & $0.31 \pm 0.04$ & $1.00 \pm 0.10$ & $1.80 \pm 0.16$ & $1.86 \pm 0.17$ \\
\hline 22 & G000.066 - 00.079 & 0.066 & -0.079 & 0.068 & -0.076 & 41 & 31 & 106 & 79 & $2.20 \pm 0.16$ & $6.92 \pm 0.45$ & $11.24 \pm 0.72$ & $15.98 \pm 1.03$ \\
\hline 23 & G000.068 + 00.241 & 0.068 & 0.241 & 0.067 & 0.244 & 26 & 17 & 52 & 37 & $0.09 \pm 0.04$ & $0.25 \pm 0.08$ & $0.37 \pm 0.12$ & $0.34 \pm 0.09$ \\
\hline 24 & $\mathrm{G} 000.070+00.175$ & 0.070 & 0.175 & 0.075 & 0.173 & 47 & 38 & 159 & 94 & $0.49 \pm 0.05$ & $1.52 \pm 0.12$ & $2.71 \pm 0.20$ & $4.86 \pm 0.34$ \\
\hline 25 & G000.070 - 00.037 & 0.070 & -0.037 & 0.071 & -0.035 & 27 & 20 & 36 & 43 & $0.29 \pm 0.06$ & $0.85 \pm 0.13$ & $1.58 \pm 0.21$ & $1.17 \pm 0.17$ \\
\hline
\end{tabular}

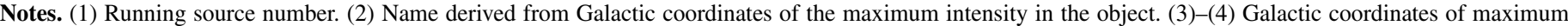

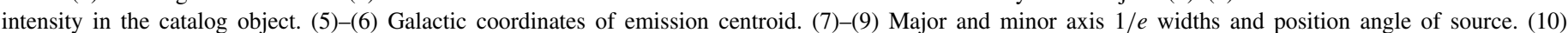

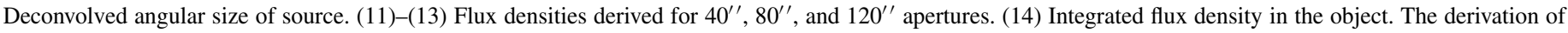
these quantities is described in Section 3.4. 


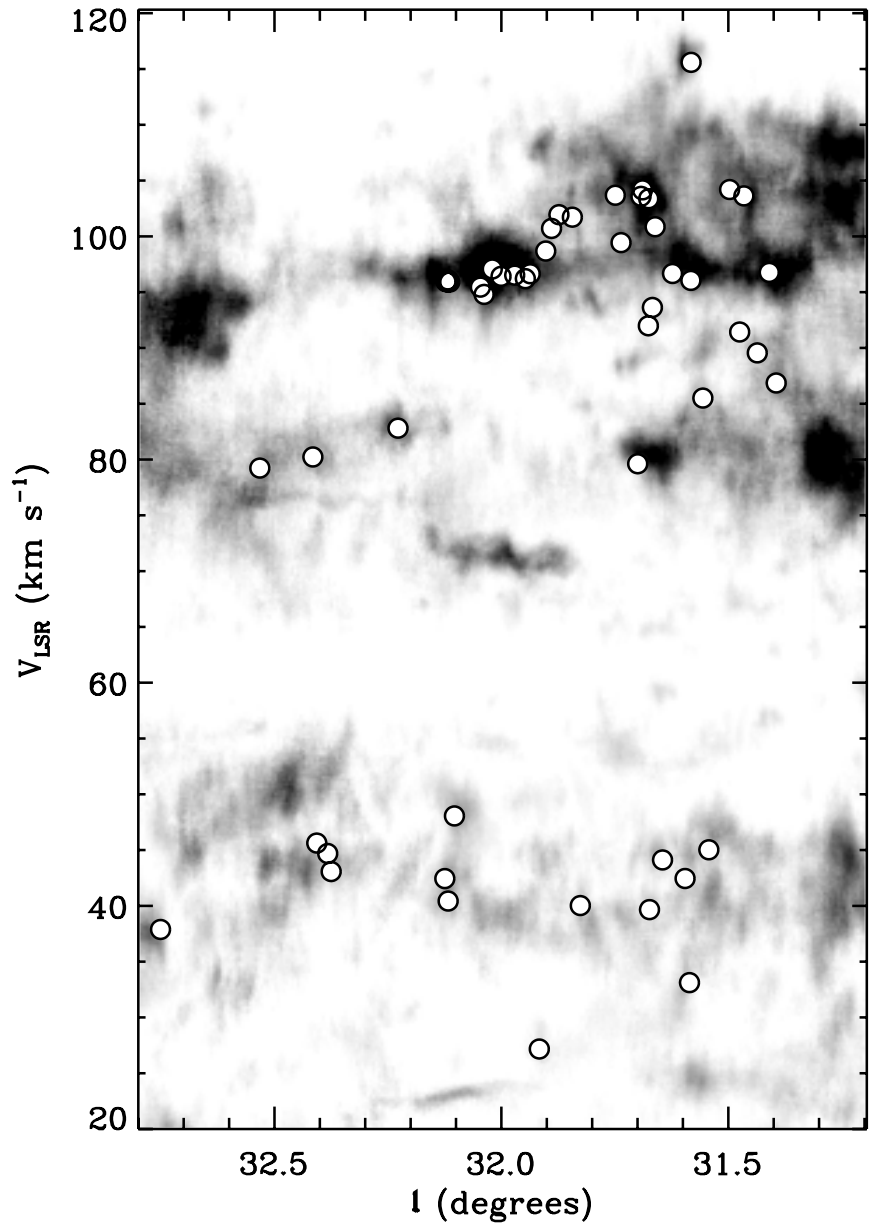

Figure 12. Latitude-integrated ${ }^{13} \mathrm{CO}(1 \rightarrow 0)$ emission with the $(\ell, V)$ locations of $42 \mathrm{BGPS} / \mathrm{NH}_{3}$ sources indicated. All BGPS sources are co-located in the data cube with significant ${ }^{13} \mathrm{CO}$ emission, though not necessarily the brightest locations.

the line properties of the ammonia spectra (i.e., $V_{\mathrm{LSR}}, \sigma_{V}, T_{K}$ ) using the model of Rosolowsky et al. (2008). Of particular note, there is only one velocity component of ammonia emission along each line of sight examined, supporting our association between BGPS emission and ammonia emission for these sources.

Using the LSR velocities of the BGPS sources, we compare these objects to the broader distribution of molecular emission in this field. In particular, we compare where these sources are found to the ${ }^{13} \mathrm{CO}(1 \rightarrow 0)$ data of Jackson et al. (2006). We present a latitude-integrated map of the molecular emission for the $\ell=31^{\circ}$ region in Figure 12. Of note in this figure, all BGPS sources are associated with the ${ }^{13} \mathrm{CO}$ emission at the $>2 \sigma$ level. However, the BGPS sources are not necessarily found at the brightest positions in the ${ }^{13} \mathrm{CO}$ data cube.

Toward these Galactic latitudes, the ${ }^{13} \mathrm{CO}$ emission is found predominantly near $V_{\mathrm{LSR}}=40 \mathrm{~km} \mathrm{~s}^{-1}$ and $90 \mathrm{~km} \mathrm{~s}^{-1}$. BGPS sources are associated with both velocity features suggesting a wide range of kinematic distances. We explore the association of BGPS emission with the tracers of low density molecular gas in the full presentation of these results (Dunham et al. 2010).

To explore the properties of BGPS sources in more detail, we estimate the source masses from the flux density of the continuum emission combined with distance and temperature estimates from the GBT data:

$$
\begin{aligned}
M & =\frac{d^{2} S}{B_{v}(T) \kappa_{v}} \\
& =13.1 M_{\odot}\left(\frac{d}{1 \mathrm{kpc}}\right)^{2}\left(\frac{S_{v}}{1 \mathrm{Jy}}\right)\left[\frac{\exp (13.0 \mathrm{~K} / T)-1}{\exp (13.0 / 20)-1}\right] .
\end{aligned}
$$

Here, we use the total flux density in the object $S$ measured in the catalog. This estimate uses a $T=20 \mathrm{~K}$ dust temperature and averages the emergent spectrum across the Bolocam band assuming the dust emissivity scales like $\lambda^{-1.8}$ and an opacity of $\kappa=0.0114 \mathrm{~cm}^{2} \mathrm{~g}^{-1}$ (Enoch et al. 2006). For these assumptions, the mean frequency of the Bolocam passband is $v=271.1 \mathrm{GHz}$. We plot the masses of the BGPS sources identified as a function of line-of-sight distance in Figure 13. For source temperatures, we use the kinetic gas temperatures derived from the ammonia observations. We use kinematic distances from the LSR velocity in the ammonia observations. Toward these Galactic longitudes, the velocity-distance relation is double valued resulting in the distance ambiguity. We plot the mass and distances derived for each source at both possible distances; so each point appears twice in the plot, once on each side of the $d=7.1 \mathrm{kpc}$ line. This $7.1 \mathrm{kpc}$ distance is determined by the tangent velocity at these longitudes. We also plot a curve representing the derived mass of the $5 \sigma$ completeness limit for point sources in this field (where $5 \sigma=0.14 \mathrm{Jy} \mathrm{beam}^{-1}$ ) with a temperature of $T=20 \mathrm{~K}$. We note that sources are not distributed uniformly down to the completeness limit since the ammonia observing targets were selected by eye and preferentially selected bright objects.

Figure 13 illustrates the wide range in possible masses extracted for the sources extracted from the survey data. First, depending on the resolution of the kinematic distance ambiguity, the masses in this field can vary by nearly an order of magnitude. Even restricting to near distances, the mass range of detected objects spans over two orders of magnitude. While a $15 M_{\odot}$ object at the low-mass range may indeed be a high-mass core, the larger mass scales at farther distances are more appropriately linked to clumps, those large structures which form stellar clusters (Williams et al. 2000). This range is largely the result of the wide range of object flux densities recovered from the original maps (see the right-hand panel of Figure 13), but naturally also depends strongly on the true distance to the source.

In Figure 14, we plot the derived values of the deconvolved projected radius $R=d \theta_{R}$ and particle density $n_{\mathrm{H}}=$ $3 M /\left(4 \pi R^{3} \mu \mathrm{m}_{\mathrm{H}}\right)$ as functions of distance. We have taken the mean particle mass to be $\mu=2.37$ (Kauffmann et al. 2008). In Figures 13 and 14, we plot the behavior of sources as a function of distance to illustrate the importance of distance determinations in describing the physical properties of these sources. In these plots, dotted lines indicate roughly the limiting sizes and densities to which the survey is sensitive. Because the ability to estimate the deconvolved source sizes depends on the signal to noise of the observations, the size limit $\theta_{R}=33^{\prime \prime}$ (= $1 \mathrm{FWHM}$ ) is chosen as a guide rather than a hard limit. It is formally possible resolve sources with sizes smaller than the beam size (Equation (6)) though the stability of such deconvolutions is suspect for $\theta_{R}<\theta_{\mathrm{bm}}$.

These plots emphasize the difference in objects recovered by the BGPS observations of the Galactic plane compared to observations of nearby molecular clouds where the Bolocam beam and sensitivity is well-matched to the size of a star-forming 

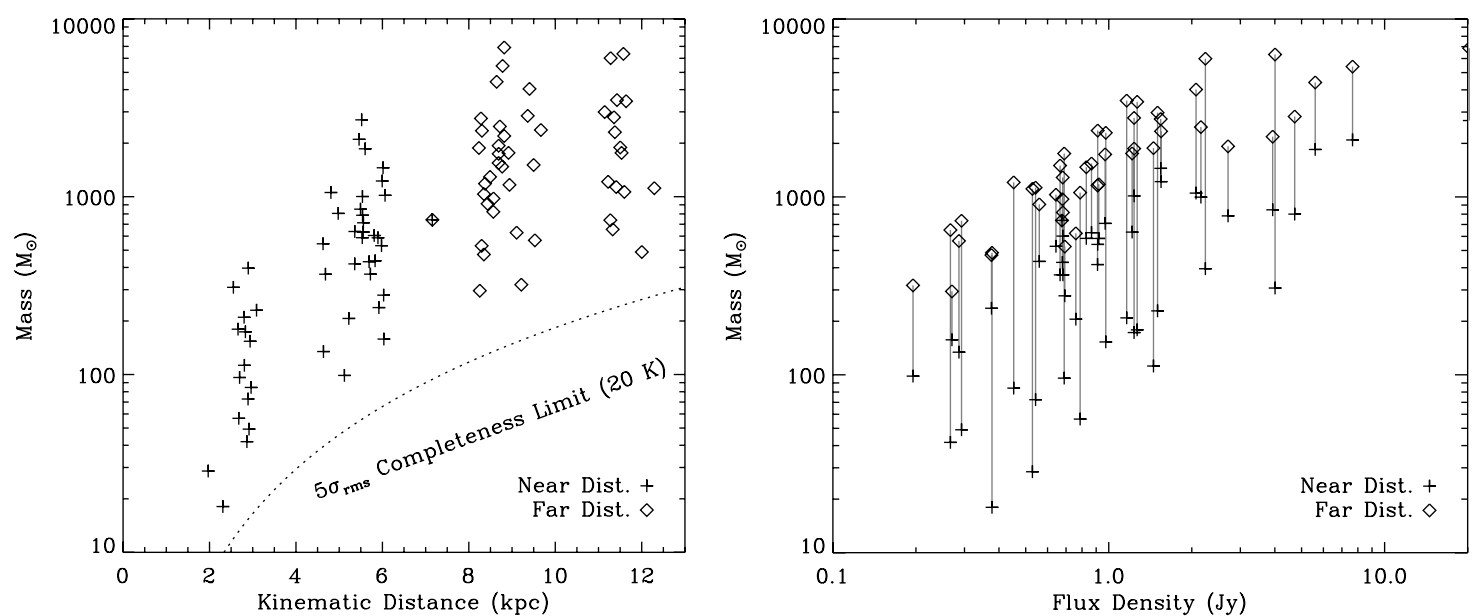

Figure 13. Mass estimates for BGPS sources as a function of line-of-sight distance (left) and flux density (right). The masses are derived from a combination of the BGPS data (for dust continuum flux densities) and ammonia data (for gas temperatures and kinematic distances) for matched sources in the $\ell=32^{\circ}$ field. This figure highlights the wide range in the masses of objects recovered in the BGPS catalog.
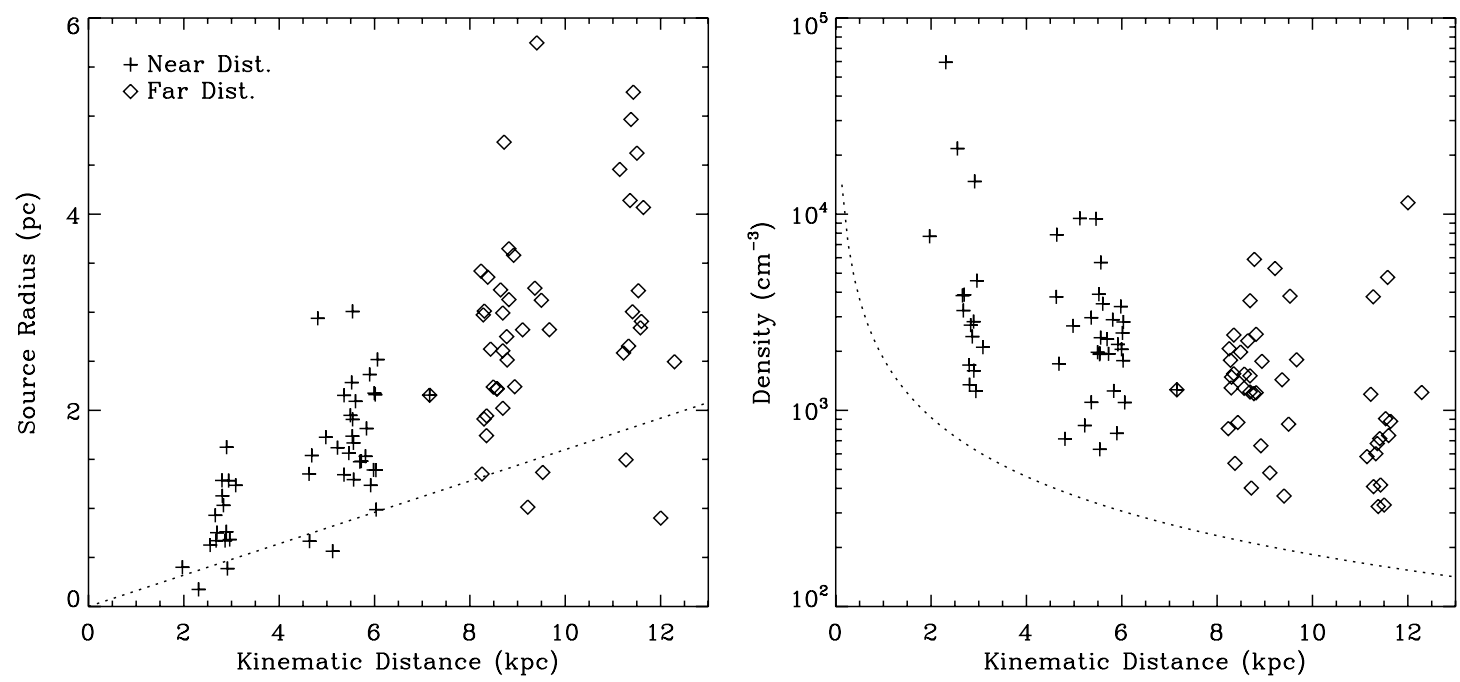

Figure 14. Radius (left) and density (right) estimates for BGPS catalog objects matched with ammonia observations in the $\ell=32^{\circ}$ field. Like Figure 13 , a given object is plotted twice for possible near and far kinematic distance estimates. The dotted line indicates the projected size for $\theta_{R}=33^{\prime \prime}$ ( 1 beam FWHM) in the left-hand panel. In the right-hand panel, the dotted line indicates the behavior for a source with $S=0.15 \mathrm{Jy}=5 \sigma$ and $\theta_{R}=33^{\prime \prime}$. Sources can appear below the $\theta_{R}=33^{\prime \prime}$ line because the beam deconvolution (Equation (6)).

core (e.g., Young et al. 2006; Enoch et al. 2006, 2007). While the BGPS catalog contains sources with core-like properties $\left(n \gtrsim 10^{4.5} \mathrm{~cm}^{-3}, R \lesssim 0.1 \mathrm{pc}\right.$ ), most of the recovered sources have larger sizes and lower density scales. The projected mass and size limits (Figure 13 (left) and Figure 14 (left)) limit the detection of individual star-forming cores in the BGPS to objects within $d \lesssim 1 \mathrm{kpc}$. Beyond this distance, BGPS is limited to the detection of clumps within larger clouds. As is illustrated by Figure 14 (right), the density of gas to which the survey is sensitive, drops off precipitously with distance to the emitting source. Objects in the catalog are thus a range of clumps and cores, and their detection depends on their distance, physical size, and dust temperature. Despite these caveats, we note that most sources in this field have mass and density scales typical of massive, dense clumps in local clouds. Given the link that these clumps have to local cluster formation, it is likely that most sources in the BGPS are associated with the formation of stellar clusters.

\section{STATISTICAL PROPERTIES OF BGPS SOURCES}

In this section, we describe the basic statistical representations of the source catalog. In all cases, the reader is cautioned that the source catalog is derived from the BGPS maps which are a filtered representation of the true sky brightness at any given position. In particular, large-scale emission is filtered out of the final maps on scales larger than 3.5 (Paper I).

\subsection{The Galactic Distribution of Flux Density}

Figure 15 presents the total flux density extracted from the images in catalog sources as a function of coordinates in the Galactic plane. In total, the catalog sources contain $11.9 \mathrm{kJy}$ of flux density. Difficulties in recovery of large-scale structure notwithstanding, this represents the emission profile of the Galaxy along the longitude and latitude direction.

The right-hand panel of Figure 15 shows that the distribution of flux density in sources peaks at the flux-weighted mean $\langle b\rangle=-0.095 \pm 0.001$. Such an offset was also seen in the ATLASGAL survey of the $12^{\circ}<b<-30^{\circ}$ region of the Galactic plane at $\lambda=870 \mu \mathrm{m}$ (Schuller et al. 2009). They speculated that the offset may result from the Sun being located slightly above the Galactic plane. To further investigate this possibility, we examined the latitudinal flux density distribution of BGPS sources as a function of longitude and compared these results to other tracers of the Galactic disk. In particular, 

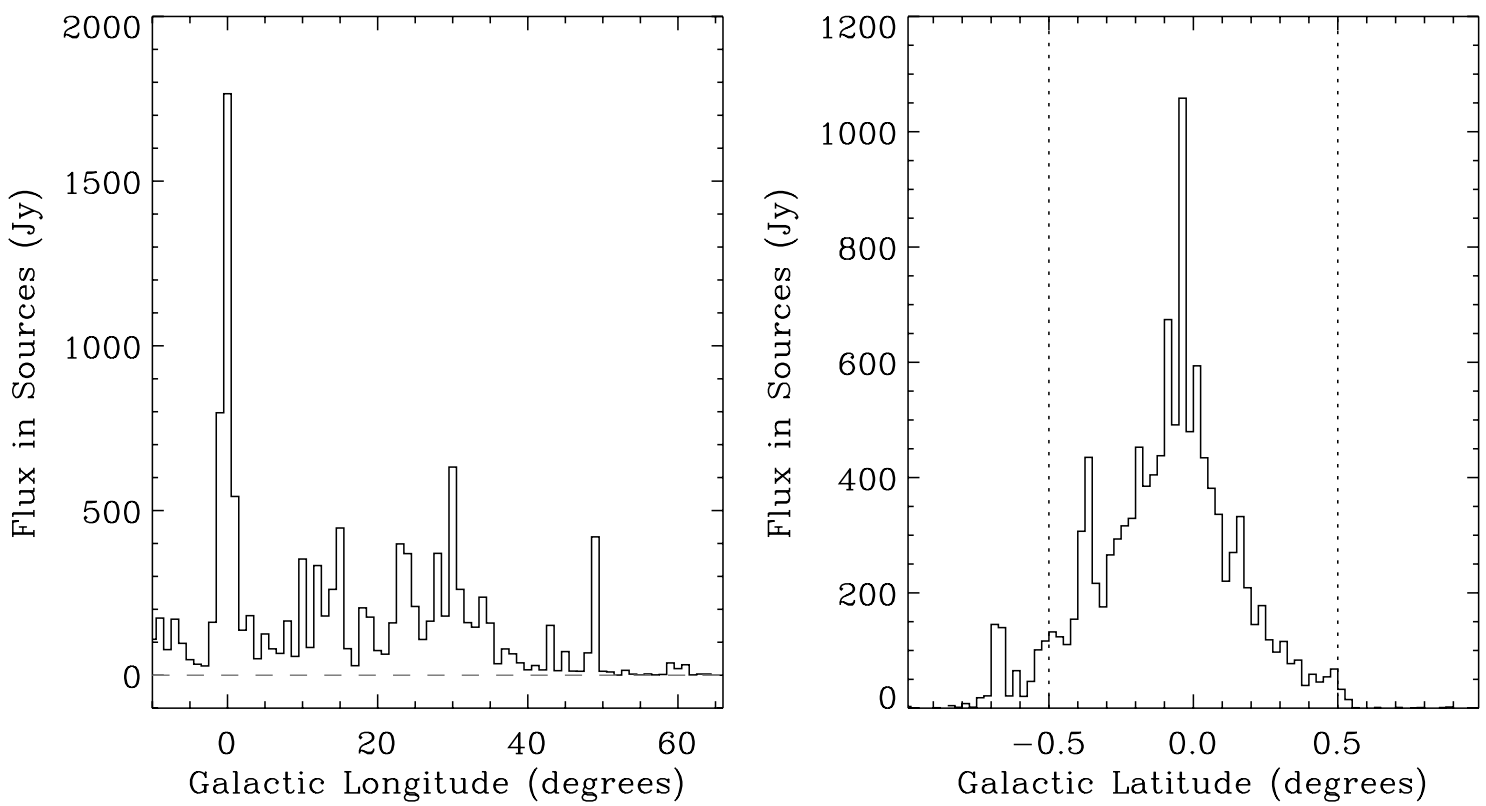

Figure 15. Longitude and latitude distribution of total flux density in catalog sources as a function of longitude and latitude in the Galactic plane. The distributions contain sources extracted in the $-10^{\circ}<\ell<66^{\circ}$ region. For $\ell>66^{\circ}$, there is minimal emission found in the map and these regions are omitted from the figure. Dotted lines in the right-hand panel indicate the rough extent of complete coverage in the latitude direction $( \pm 0.5)$.

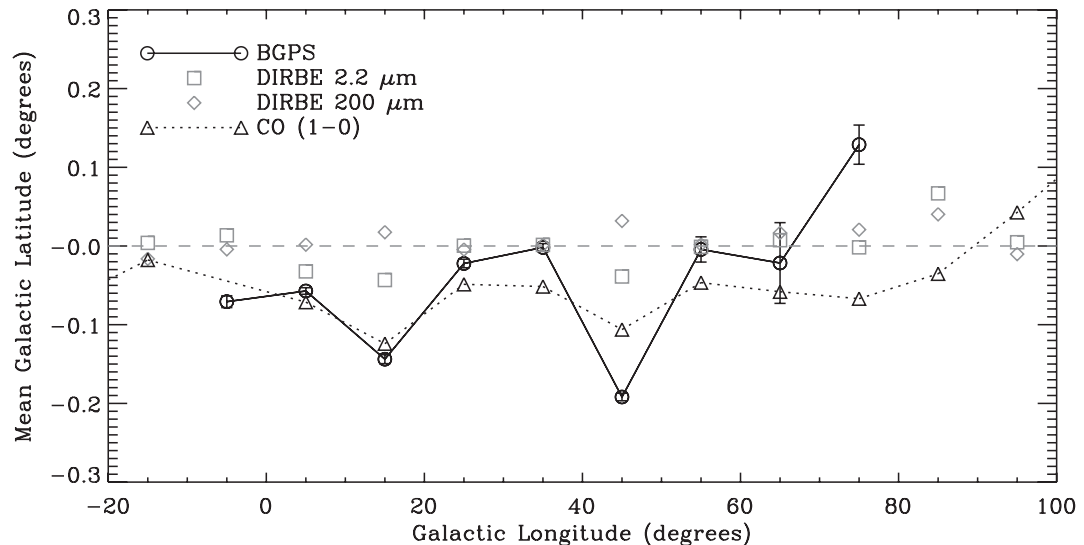

Figure 16. Mean Galactic latitude for various tracers of the disk. The BGPS catalog sources show a mean latitude of $\langle b\rangle=-0.095$ which varies significantly with Galactic longitude. Data are averaged over the range $-0.5<b<0.5$ in $10^{\circ}$ bins in longitude. The BGPS sources are most closely associated with the CO emission whereas tracers of the stellar population (DIRBE $2.2 \mu \mathrm{m}$ ) and warm dust (DIRBE $200 \mu \mathrm{m}$ ) show less offset toward negative latitudes.

we compared the flux-density-weighted values of the mean latitude for objects in our catalog to the COBE/DIRBE data at $\lambda=2.2 \mu \mathrm{m}$ and $200 \mu \mathrm{m}$ (Hauser et al. 1998) and the integrated ${ }^{12} \mathrm{CO}(1-0)$ data of Dame et al. (2001). The results of the comparison are shown in Figure 16. For all data sets, we only compute the flux-density-weighted average latitude over the range $-0.5<b<0.5$ which is the latitude range over which the BGPS is spatially complete. The data are averaged in $10^{\circ}$ bins. The offset of BGPS sources appears at nearly all Galactic longitudes, but is most pronounced toward the Galactic center $\left(-10^{\circ}<\ell<25^{\circ}\right)$. The $\mathrm{CO}(1-0)$ data follow a similar trend with a significant offset toward negative latitudes. We verified that the effects persist in the $\mathrm{CO}$ data if the averaging range is expanded to larger latitudes (foreground emission from the Aquila rift affects the averaging for large latitude ranges $|b|<5^{\circ}$ ). We also note that the emission profiles from the DIRBE data do not follow the offset trends seen in the millimeter continuum or the CO. The DIRBE $2.2 \mu \mathrm{m}$ data primarily traces emission from stellar photospheres and is affected by dust extinction and the $200 \mu \mathrm{m}$ traces warm dust emission. Both of these emission features show a more symmetrical distribution around the Galactic midplane than do those tracers associated with the molecular gas. We conclude that the offsets toward negative latitude are primarily associated with the molecular ISM. The significant variations with the mean offset are likely the result of individual star-forming structures rather than a global property of all Galactic components. For example, the large negative excursion in the $40^{\circ}<\ell<50^{\circ}$ bin is due to the W51 star formation complex at $\left(49^{\circ} .5,-0.4\right)$. At $\ell>60^{\circ}$, the space density of BGPS sources drops sharply, so the effects are dominated by the particular distribution of the dense gas within the molecular complex. Specifically, in the $\ell \sim 70^{\circ}$ region, there are more dense gas structures at $b>0^{\circ}$ in the Cygnus $\mathrm{X}$ and Cygnus OB 7 region than there are at $b<0^{\circ}$, while the lower density molecular gas does not exhibit this small-number effect.

\subsection{The Flux Density Distribution of BGPS Sources}

In Figure 17, we present two representations of the flux density distribution for objects in the BGPS catalog. The flux 

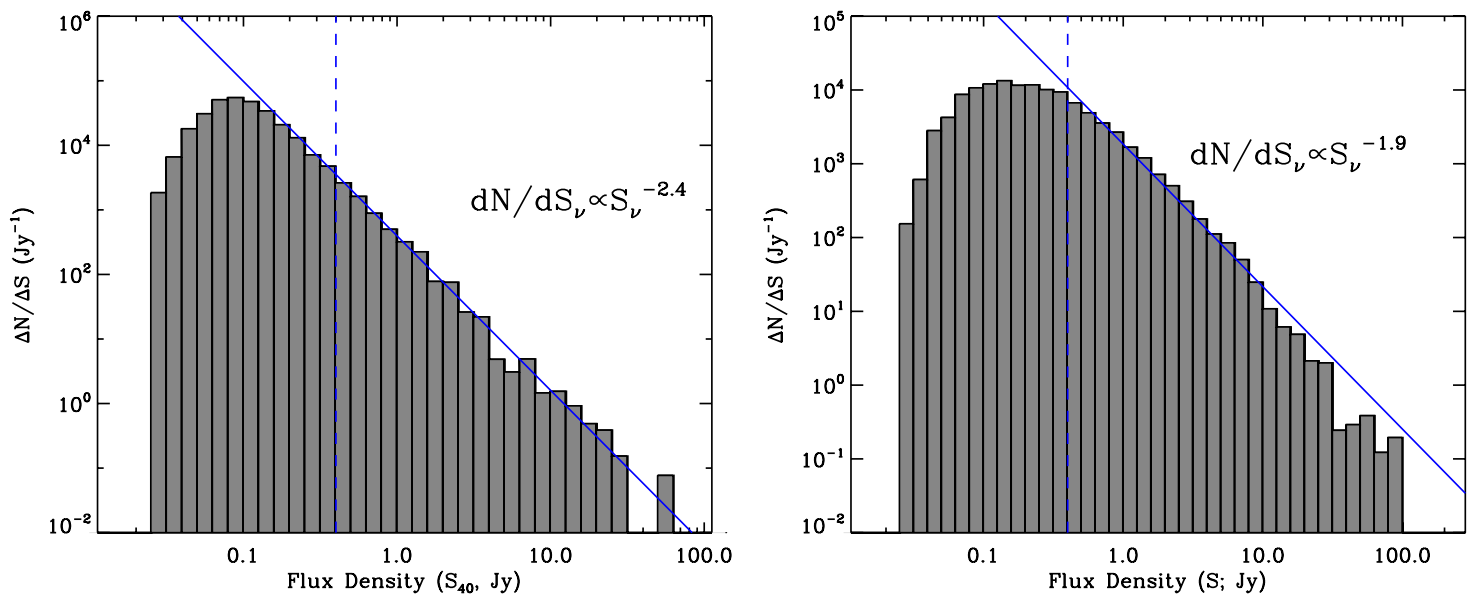

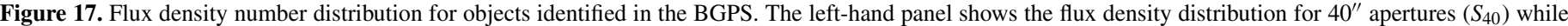

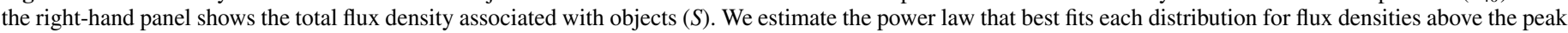

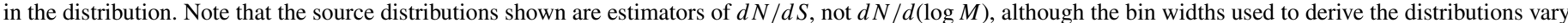
logarithmically.

(A color version of this figure is available in the online journal.)

density distribution in $40^{\prime \prime}$ apertures is shown in the left-hand panel and follows a power-law form over nearly three orders of magnitude in flux density from the point source completeness limit to the upper flux density limit in the survey. We have fit a power law to the approximation to the differential flux density spectrum $d N / d S \approx \Delta N / \Delta S \propto S^{-\alpha}$ and find that an index $\alpha=2.4 \pm 0.1$ represents the data quite well. Despite the similarity to the Salpeter (Salpeter 1955) index $\alpha=2.35$, we suspect that this is coincidence because the BGPS sources are found at a wide variety of distances. This range of distances necessitates a complete treatment of the conversion of flux density to mass. Indeed, the Balloon-borne Large Aperture Submillimeter Telescope (BLAST) survey of the Vela molecular complex at $\lambda=250,350$, and $500 \mu \mathrm{m}$ found a marginally steeper distribution of object mass- $d N / d M \propto M^{-2.8 \pm 0.2}$ for cold cores-than the BGPS finds for flux density (Netterfield et al. 2009). A careful treatment of the distances to BGPS sources may find the mass distribution to be comparable between BGPS objects and those found in the BLAST survey.

In the right panel of Figure 17, we plot the differential flux density distribution for the total flux density in the objects $S$ (Equation (7)) and find that a power law is not as good a representation for the catalog. Even though the completeness limits should be the same for both flux density measurements, the power-law behavior for the total flux density only become apparent above a much higher flux density level of $0.7 \mathrm{Jy}$ where $\alpha=1.9 \pm 0.1$. The difference between these two limits arises entirely because of the extended structure accounted for in the total flux density measurement. A detection near the completeness limit is necessarily similar to a point source. To detect extended objects with any degree of fidelity requires a much higher peak value of the flux density so that connected, extended structure is apparent. Thus, source with flux densities near the completeness limit will not sample the full range of source sizes that brighter objects will. Hence, the detection of extended structure is only apparent in bright sources leading to a higher limit. We also note that a power-law index fit to the distribution above the limit of 0.7 Jy gives a significantly different value compared to the index for flux densities derived for $40^{\prime \prime}$ apertures. This simply reflects the additional effects of the included sizes of the sources. In general, sources with bright peaks (measured in the $40^{\prime \prime}$ apertures) also tend to be large

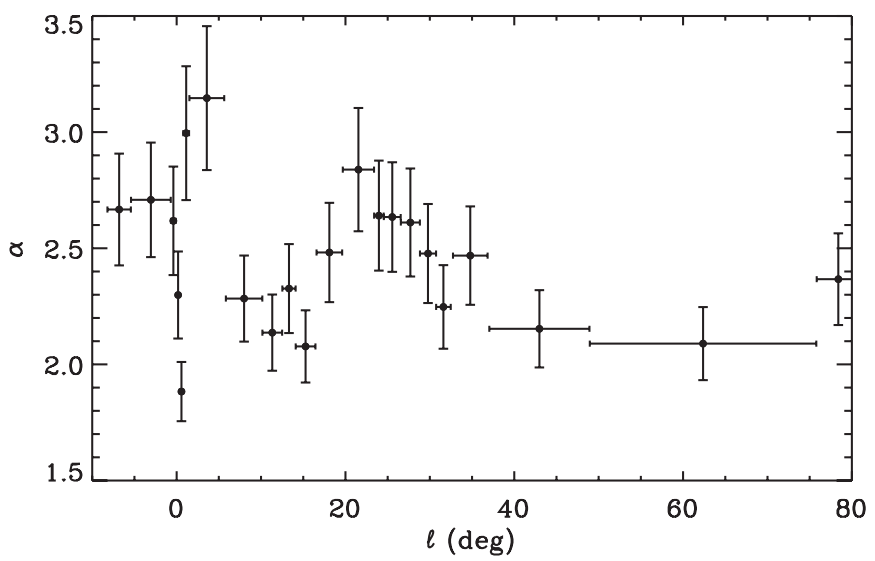

Figure 18. Power-law index on the flux density distribution as a function of Galactic longitude, derived for groups of 50 sources above the survey completeness limit of $0.4 \mathrm{Jy}$. The flux densities are measured in $40^{\prime \prime}$ apertures across the Galactic plane.

so their total recovered flux density is larger than for smaller sources. This effectively makes the distribution more top-heavy, reducing the magnitude of the exponent.

\subsection{Variations in the Flux Density Distribution}

In Figure 18, we show the value derived for the slope of the flux density distribution as a function of Galactic latitude over the contiguous region of the BGPS. We divide the catalog into groups of 50 sources based on the galactic longitude above the survey completeness limit of $0.4 \mathrm{Jy}$. The variation of $\alpha$ scatters around the global average of $\alpha=2.4$ with marginally significant variation as a function of longitude. The variations are strongest in regions of high source density suggesting that blending may contribute to the derived distribution. Specifically, the algorithm has a native angular scale over which it searches for local maxima (a box with edge length of 2 beam FWHM). Structures tend to be subdivided on scales near this search scale. Large, bright objects in crowded regions of the plane may be subdivided more often than sources in less crowded regions. Indeed, in the $\ell=60^{\circ}$ region, where the source density distribution is lowest, the flux density distribution is the most shallow. 

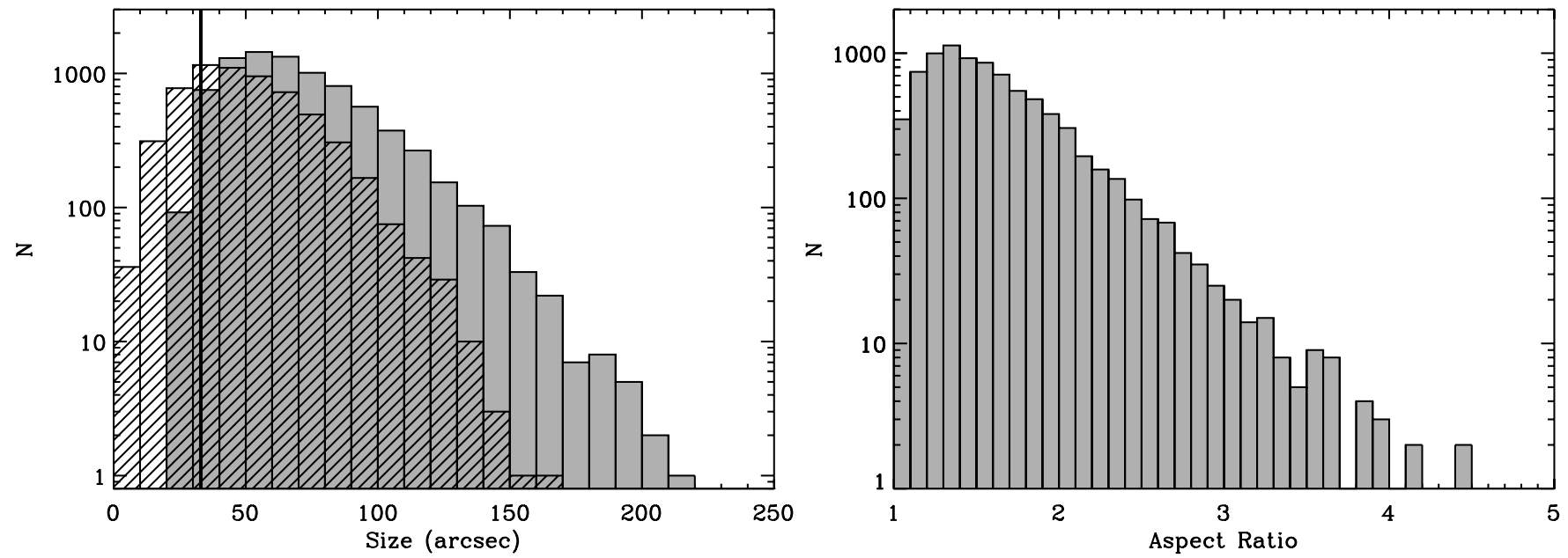

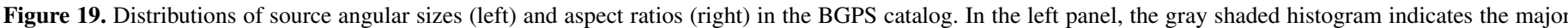

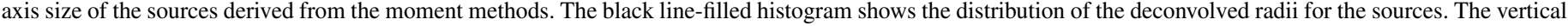
line is plotted at the FWHM of the beam. In the right panel, the distribution plots the major-to-minor aspect ratio for the moments of the source.

\subsection{Size Distribution of Sources}

The measured sizes of sources varies significantly for the BGPS sources. We plot the distribution of source sizes and aspect ratios in Figure 19 for the catalog sources. We show the major axis size $\left(\eta \sigma_{\text {maj }}\right.$ where $\left.\eta=2.4\right)$ determined from the moment method and the deconvolved angular radius of the source $\left(\theta_{R}\right)$, which includes both major and minor axes (Equation (6)). Because of the deconvolution, the radius $\theta_{R}$ can be smaller than the beam size. However, we also note that some of the major axis sizes are also smaller than the beam size. This results from applying moment methods without accounting for all emission down to the $I=0$ level. The clip at $1 \sigma$ truncates the low-significance band of emission around the sources. For faint sources, the truncation will clip a significant fraction of the emission resulting in an underestimate of the source size. While this effect can be corrected for, we refrain from doing so since such corrections require extrapolating the behavior of the sources below the noise floor in the images.

We also present data for the aspect ratios of the catalog sources defined as $\sigma_{\text {maj }} / \sigma_{\min }$. Both the radii and the aspect ratios show a peaked distribution with an exponential decline. The typical source in the BGPS has a major axis size of $60^{\prime \prime}$ and a minor axis size of $45^{\prime \prime}$. However, there are significant numbers of large-aspect-ratio sources which represents asymmetric clump structure and, for faint sources, the prevalence of filamentary structure in the survey.

\section{SUMMARY}

We present a catalog of 8358 sources extracted from the initial release of the BGPS (Aguirre et al. 2009). The BGPS is a survey of the Galactic Plane from $-10^{\circ}<\ell<90.5$ with extensions into the outer Galaxy. The catalog is generated with an automated source extraction algorithm, Bolocat, that is designed for wide-field Bolocam mapping. The algorithm mimics a by-eye identification of sources in the BGPS. The catalog products are formulated to allow easy follow-up since many of the objects in the BGPS are relatively unexplored.

The Bolocat algorithm identifies sources based on their significance with respect to a local estimate of the noise in the BGPS maps. Regions with high significance are then subdivided into individual sources based on the presence of local maxima within the region. Each pixel in the BGPS image is assigned to at most one catalog source using a seeded watershed, similar to the Clumpfind (Williams et al. 1994) or Source EXtractor algorithms (Bertin \& Arnouts 1996). The properties of the BGPS sources are measured using the moments of the images for each of the assigned sources.

We have conducted tests of the Bolocat algorithm using artificial sources injected into observations in the survey fields. The Bolocat algorithm extracts sources with a 99\% completeness limit of $5 \sigma$ where $\sigma$ is the local rms of the noise. Since the noise varies across the BGPS fields, the $98 \%$ completeness limit for the survey is $0.4 \mathrm{Jy}$, set by the highest noise fields.

Using spectroscopic observations of the $\mathrm{NH}_{3}(1,1)$ inversion transition, we have characterized several of the BGPS sources near $\ell=32^{\circ}$. We find that the objects in the catalog are likely best described as clumps (Williams et al. 2000), though there is substantial variation in the properties of the sources. Since the catalog is generated using a significance threshold, any source that produces a signal $>5 \sigma$ in the $33^{\prime \prime}$ beam size of the instrument will be detected. Hence, both nearby, small, low-mass objects and distant, large, high-mass objects can be detected with similar flux densities.

We find the flux density distribution of sources in the BGPS follows a power-law form. The flux densities of objects extracted in a $40^{\prime \prime}$ aperture follows a power law over nearly three orders of magnitude: $d N / d S_{40} \propto S_{40}^{-2.4 \pm 0.1}$. We also find that the mean Galactic latitude of objects in the survey lies at $\langle b\rangle=-0.095 \pm 0.001$, with some variation across the survey region according to the presence of large star-forming complexes.

The BGPS is supported by the National Science Foundation through the NSF grant AST-0708403. E.R. acknowledges partial support from an NSF AAP Fellowship (AST-0502605) and a Discovery Grant from NSERC of Canada. N.J.E. and M.K.N. acknowledge support from the NSF grant AST-0607793. We acknowledge the cultural role and reverence that the summit of Mauna Kea has within the Hawaiian community. We are fortunate to conduct observations from this mountain. The Green Bank Telescope is operated by the National Radio Astronomy Observatory. The National Radio Astronomy Observatory is a facility of the National Science Foundation operated under cooperative agreement by Associated Universities, Inc.

Facilities: CSO (Bolocam); GBT (K-band/ACS) 


\section{REFERENCES}

Aguirre, J., et al. 2009, ApJ, submitted

Bally, J., et al. 2009, ApJ, submitted

Bertin, E., \& Arnouts, S. 1996, A\&AS, 117, 393

Dame, T. M., Hartmann, D., \& Thaddeus, P. 2001, ApJ, 547, 792

Dougherty, E. 1992, An Introduction to Morphological Image Processing (Bellingham, WA: SPIE Optical Engineering Press)

Dunham, M. K., et al. 2010, ApJ, submitted

Enoch, M. L., Glenn, J., Evans, N. J., Sargent, A. I., II, Young, K. E., \& Huard, T. L. 2007, ApJ, 666, 982

Enoch, M. L., et al. 2006, ApJ, 638, 293

Evans, N. J., II 1999, ARA\&A, 37, 311

Friesen, R. K., Di Francesco, J., Shirley, Y. L., \& Myers, P. C. 2009, ApJ, 697, 1457

Glenn, J., et al. 2003, Proc. SPIE, 4855, 30

Hauser, M. G., et al. 1998, ApJ, 508, 25

Jackson, J. M., et al. 2006, ApJS, 163, 145

Johnstone, D., Wilson, C. D., Moriarty-Schieven, G., Joncas, G., Smith, G., Gregersen, E., \& Fich, M. 2000, ApJ, 545, 327

Kauffmann, J., Bertoldi, F., Bourke, T. L., Evans, N. J., II, \& Lee, C. W. 2008, A\&A, 487, 993
Moore, T. J. T., Shipman, R. F., Plume, R., Hoare, M. G., \& Jps International Collaboration, 2005, in Protostars and Planets V, ed. B. Reipurth, D. Jewitt, \& K. Keil (Tucson, AZ: Univ. Arizona Press), 8370

Motte, F., Andre, P., \& Neri, R. 1998, A\&A, 336, 150

Netterfield, C. B., et al. 2009, ApJ, 707, 1824

Noriega-Crespo, A., \& Molinari, S. 2008, in BAAS, 40, 271

Press, W. H., Teukolsky, S. A., Vetterling, W. T., \& Flannery, B. P. 1992, Numerical Recipes in C, The Art of Scientific Computing (2nd ed.; Cambridge: Cambridge Univ. Press)

Reid, M. J., et al. 2009, ApJ, 700, 137

Rosolowsky, E. W., Pineda, J. E., Foster, J. B., Borkin, M. A., Kauffmann, J., Caselli, P., Myers, P. C., \& Goodman, A. A. 2008, ApJS, 175 , 509

Salpeter, E. E. 1955, ApJ, 121, 161

Schuller, F., et al. 2009, A\&A, 504, 415

Williams, J. P., Blitz, L., \& McKee, C. F. 2000, in Protostars and Planets IV, ed. V. Mannings, A. P. Boss, \& S. S. Russell (Tucson, AZ: Univ. Arizona Press), 97

Williams, J. P., de Geus, E. J., \& Blitz, L. 1994, ApJ, 428, 693

Yoo, T., (ed.) 2004, Insight Into Images Principles and Practice for Segmentation, Registration and Image Analysis (Wellesley, MA: A K Peters)

Young, K. E., et al. 2006, ApJ, 644, 326 